FSU-HEP-981015

UH-511-925-99

NU-TH-99-50

\title{
THE REACH OF FERMILAB TEVATRON UPGRADES FOR SU(5) SUPERGRAVITY MODELS WITH NON-UNIVERSAL GAUGINO MASSES
}

\author{
Greg Anderson ${ }^{1}$, Howard Baer ${ }^{2}$, Chih-Hao Chen ${ }^{2}$ and Xerxes Tata ${ }^{3}$ \\ ${ }^{1}$ Department of Physics, Northwestern University, Evanston IL 60208 USA \\ ${ }^{2}$ Department of Physics, Florida State University, Tallahassee, FL 32306 USA \\ ${ }^{3}$ Department of Physics and Astronomy, University of Hawaii, Honolulu, HI 96822 USA
}

(August 8, 2018)

\begin{abstract}
We explore the reach of luminosity upgrades of the Fermilab Tevatron collider for $S U(5)$ supergravity models in which non-universal GUT-scale gaugino masses arise via a vacuum expectation value for the auxiliary component of a superfield that transforms as a 24, 75 or 200 dimensional representation of $S U(5)$. This results in a different pattern of sparticle masses and mixing angles from what is expected in the minimal supergravity model (mSUGRA) with universal GUT scale gaugino masses. We find that the resulting signal cross sections, and hence the reach of the Tevatron, are sensitive to the gaugino masses at the GUT scale. In the $\mathbf{2 4}$ model, the large splitting amongst the two lightest neutralinos leads to SUSY events containing many isolated leptons, including events with a real leptonic $Z$ boson plus jets plus missing energy signal which is visible over much of parameter space. In contrast, in the $\mathbf{7 5}$ and $\mathbf{2 0 0}$ models, the reach via leptonic SUSY signals is greatly reduced relative to mSUGRA, and the signal is usually visible only via the canonical $E_{T}+$ jets channel.
\end{abstract}

PACS numbers: 14.80.Ly, 13.85.Qk, 11.30.Pb

Typeset using REVTEX 


\section{INTRODUCTION}

The minimal supergravity model (mSUGRA) [1] provides a well-motivated and economical framework in which to embed the Minimal Supersymmetric Standard Model [2], or MSSM. In mSUGRA, supersymmetry is broken in a hidden sector, and SUSY breaking is communicated to the visible sector MSSM fields via interactions of gravitational strength. Motivated by the apparently successful gauge coupling unification in the MSSM, it is usually assumed that this leads to a common value $m_{0}$ for all scalars, a common mass $m_{1 / 2}$ for all gauginos, and a common trilinear SUSY breaking term $A_{0}$ at the scale $M_{G U T} \simeq 2 \times 10^{16} \mathrm{GeV}$. The soft SUSY breaking terms, the gauge and Yukawa couplings and the supersymmetric $\mu$ term are all then evolved from $M_{G U T}$ to some scale $M \simeq M_{\text {weak }}$ using renormalization group equations (RGE's). Electroweak symmetry is broken radiatively due to the large top quark Yukawa coupling. The resulting weak scale spectrum of superpartners and their couplings can then be derived in terms of four continuous plus one discrete parameters

$$
m_{0}, m_{1 / 2}, A_{0}, \tan \beta \text { and } \operatorname{sgn}(\mu),
$$

in addition to the usual parameters of the standard model.

In studies of mSUGRA and other supersymmetric extensions of the standard model based on gauge-unification and the gravitational mediation of supersymmetry-breaking, it is often assumed (as discussed above) that the unification of gauge interactions implies a similar unification of gaugino masses at the scale of gauge-coupling unification. However, gravitationally mediated supersymmetry breaking may lead to non-universal gaugino masses even in the presence of gauge coupling unification. We present a class of models which contain non-universal gaugino masses, discuss their experimental signatures at the Fermilab Tevatron, and contrast those signatures with those of mSUGRA. The models we discuss represent equally predictive alternatives to the canonical universal gaugino mass scenario.

If gravity is the messenger which communicates supersymmetry breaking from the hidden to the visible sector, supersymmetry breaking mass terms for gauginos can arise from higher dimensional interactions which couple a chiral superfield to the supersymmetric field strength [3]. These interactions arise from the locally supersymmetric gauge field strength interactions:

$$
\mathcal{L} \supset \int d^{4} \theta E\left(R^{-1} f_{a b} W^{a} W^{b}+h . c\right)
$$

with a gauge kinetic function $f_{A B}=\delta_{A B}+\Phi_{A B} / M_{\text {Planck }}+\ldots$. The fields $\Phi_{A B}$ transform as left handed chiral superfields under supersymmetry transformations, and as the symmetric product of two adjoints under gauge symmetries. The lowest order contribution to gaugino masses arising from the interaction above comes from a dimension five operator:

$$
\mathcal{L} \supset \int d^{2} \theta W^{a} W^{b} \frac{\Phi_{a b}}{M_{\text {Planck }}}+\text { h.c. } \supset \frac{\left\langle F_{\Phi}\right\rangle_{a b}}{M_{\text {Planck }}} \lambda^{a} \lambda^{b}+\ldots,
$$

where the $\lambda^{a, b}$ are the gaugino fields, and $F_{\Phi}$ is the auxillary field component of $\Phi$.

In conventional models of supersymmetry breaking, the fields $F_{\Phi}$ which break supersymmetry are treated as gauge singlets. However, in principle, the chiral superfield which communicates supersymmetry breaking to the gaugino fields can lie in any representation 
found in the symmetric product of the adjoint. Non gauge singlet vacuum expectation values for the supersymmetry preserving component of $\Phi_{A B}$ have been considered previously [4,5] for their perturbative effect on gauge coupling unification and also for their effect on gaugino masses [4,6 8]. Here we consider the effect of supersymmetry breaking vacuum expectation values of $\Phi_{A B}$ which lead to maximally predictive gaugino masses. In the context of $S U(5)$ grand unification, $F_{\Phi}$ belongs to an $S U(5)$ irreducible representation which appears in the symmetric product of two adjoints:

$$
(24 \times 24)_{\text {symmetric }}=1 \oplus \mathbf{2 4} \oplus \mathbf{7 5} \oplus \mathbf{2 0 0},
$$

where only 1 yields universal masses. Only the component of $F_{\Phi}$ that is 'neutral' with respect to the SM gauge group should acquire a vaccuum expectation value (vev), $\left\langle F_{\Phi}\right\rangle_{a b}=c_{a} \delta_{a b}$, with $c_{a}$ then determining the relative magnitude of the gaugino masses at $M_{G U T}$. The relations amongst the various GUT scale gaugino masses have been worked out, e.g. in Ref. [8]. The relative $G U T$ scale $S U(3), S U(2)$ and $U(1)$ gaugino masses $M_{3}, M_{2}$ and $M_{1}$

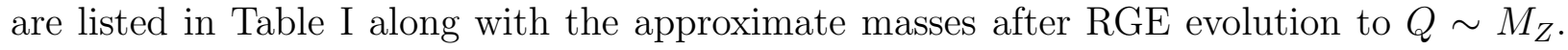
Here, motivated by the measured values of the gauge couplings at LEP, we assume that the vev of the scalar component of $\Phi$ is neglible. In principle, as shown in Fig. [1, an arbitrary linear combination of the above irreducible representations is also allowed. We consider the implications of models where the dominant contribution to gaugino masses arises from a single irreducible representation. 7 Each of the three non-singlet models is as predictive as the canonical singlet case, and all are compatible with the unification of gauge couplings. These scenarios represent the predictive subset of the more general case [7] of an arbitrary superposition of these representations $\mathbb{A}^{2}$, the most interesting being a superposition of gauge singlet and adjoint fields.

As we discuss in Section III, signals of supersymmetry- and hence the reach of the Tevatron- is sensitive to the structure of the gaugino masses as the GUT scale. The reach of the Fermilab Tevatron collider for mSUGRA models with universal gaugino masses has been worked out for both low [9 12] as well as high [13] values of the parameter $\tan \beta$. For low values of $\tan \beta$ and high integrated luminosity, the clean trilepton signal (C3L) [14] from $\widetilde{W}_{1} \widetilde{Z}_{2} \rightarrow \ell^{\prime} \bar{\ell} \ell+\mathbb{E}_{T}$ usually offers the best prospect for a SUSY discovery. For parts of parameter space, a SUSY signal might be found in several different channels. For large

\footnotetext{
${ }^{1}$ From the point of view of the theory below the GUT scale, we may consider the use of the large representations listed in Eq. (1.4) as a calculational convenience. Only the $S U(3) \times S U(2) \times U(1)$ singlet components of these representations are relevant to our discussion. The remaining states may obtain masses which are heavy compared to $M_{G U T}$. Any relic of a large GUT representation which survives below the GUT scale and has a non-vanishing coupling to the supersymmetric field strength, must lie in the symmetric product the adjoint representations of the unified group, and further this relic must be a $S U(3) \times S U(2) \times U(1)$ singlet. For the unification group $S U(5)$, the complete set of masses produced by relics from pure $S U(5)$ representations are those listed in Table 1.

${ }^{2}$ In Ref. [6], a specific linear combination was fixed by the additional assumption of the vanishing of this contribution to leptoquark gaugino masses.
} 
$\tan \beta$, discovery via the C3L signal becomes increasingly difficult because sparticle decays to $\tau$-leptons and $b$-quarks becomes enhanced relative to decays to $e$ 's and $\mu$ 's, but the range of parameters over which the signal is observable may be extended by the use of softer cuts on the leptons as emphasized by Barger et al. [15].

We should add that we do not specially advocate any particular representation for $\left\langle F_{\Phi}\right\rangle$ on theoretical grounds. Our main motivation is to examine the sensitivity of the various signals via which SUSY might manifest itself at future runs of the Tevatron to changes in the underlying framework. It is especially important to do so when assessing the search capabilities of future facilities, particularly because we do not as yet have a dynamical understanding of SUSY breaking, which can affect the phenomenology via the pattern of sparticle masses and mixing angles.

With this in mind, the event generator ISAJET [16] (versions $\geq 7.37$ ) has been upgraded to accommodate SUGRA models with various non-universal soft SUSY breaking terms. In this study, we use ISAJET to simulate models with non-universal gaugino mass parameters at the scale $M_{X}$ assuming universality of other parameters. The model parameter space used in this paper thus corresponds to

$$
m_{0}, M_{3}^{0}, A_{0}, \tan \beta \text { and } \operatorname{sgn}(\mu),
$$

where $M_{i}^{0}$ is the $S U(i)$ gaugino mass at scale $Q=M_{G U T} . M_{2}^{0}$ and $M_{1}^{0}$ can then be calculated in terms of $M_{3}^{0}$ according to Table $\square$. ISAJET calculates an iterative solution to the 26 RGEs, and imposes the radiative electroweak symmetry breaking constraint. This determines all sparticle masses and mixings. Next, branching fractions for all sparticles, particles and Higgs bosons are calculated. Supersymmetric particle production events can be generated for all possible $2 \rightarrow 2$ SUSY hard scattering subprocesses. Sparticle production is followed by initial and final state parton showers, cascade decays, hadronization and underlying event simulation. Thus, specific assumptions about soft SUSY breaking terms that are motivated by GUT or String scale physics can be directly tested at collider experiments.

In this paper, we explore the consequences of non-universal gaugino masses for $S U(5)$ SUGRA GUT models for the Fermilab Tevatron collider and its planned upgrades. Our goals are several.

- We wish to establish the capability of the Tevatron and its upgrades to discover or rule out SUSY within the context of models alternative to mSUGRA. The set of models we examine maintain many of the attractive features of generic SUGRA models, while exhibiting radically different sparticle mass spectra and mixing angles from the commonly examined models which assume universality.

- We want to see if this class of models examined can be distinguished one from another. If certain SUSY signals are observed, the answer appears to to be yes for a limited region of model parameter space.

- Are there any new signals for SUSY that can occur within the context of non-standard SUSY models? We will see that in the $F_{\Phi} \sim \mathbf{2 4}$ model, there is a large range of parameter space that leads to signal events containing real leptonic $Z$ bosons. These signals occur much more rarely in the mSUGRA model. 
Non-universality of gaugino masses can also arise in other model contexts [17 including some string models [18]. Phenomenological consequences of O-II string models have been examined in Ref. [19.

In Sec. II, we outline features of the mass spectra that are consequences of the assumptions about the $S U(5)$ representation of the hidden sector field(s) $\Phi$ that can occur. In Sec. III, we outline the various types of signals that could occur for SUSY models, and our signal and background event generator calculations. In Sec. IV, we present results of the reach of Tevatron upgrade options for each of the four models considered. In Sec. V, we present a summary and some conclusions.

\section{SPARTICLE MASSES FOR SUGRA MODELS}

We begin by illustrating the evolution of the magnitude of soft SUSY breaking masses versus scale $Q$ in Fig. 2 for the four model choices a) $\left.\left.F_{\Phi} \sim \mathbf{1}, b\right) F_{\Phi} \sim \mathbf{2 4}, c\right) F_{\Phi} \sim 75$ and d) $F_{\Phi} \sim 200$. We take $m_{0}=100 \mathrm{GeV}, M_{3}^{0}=125 \mathrm{GeV}, A_{0}=0, \tan \beta=5$ and $\mu>0$. Throughout this paper, we take $m_{t}=175 \mathrm{GeV}$.

The gaugino masses are denoted by dashed lines, while Higgs masses are denoted by dotted lines and squark and slepton masses are denoted by solid lines. For the usual mSUGRA case illustrated in Fig. 2 $a$, the gaugino masses evolve from a common GUT scale value. For the $F_{\Phi} \sim \mathbf{2 4}$ model in frame $b$ ), the splitting in GUT scale gaugino masses shown in Table I leads to a large mass gap between $M_{1}$ and $M_{2}$ at the weak scale, and also a large mass gap between left and right sfermions. In case $c$ ) for $F_{\Phi} \sim 75$, the large GUT scale splitting of gaugino masses leads to near gaugino mass degeneracy at the weak scale, and also similar masses for both squarks and sleptons. Finally, for case $d$ ) with $F_{\Phi} \sim \mathbf{2 0 0}$, the large GUT scale splitting leads to $M_{2}, M_{3}<M_{1}$ at the weak scale. In addition, the large GUT scale values of $M_{1}$ and $M_{2}$ cause the weak scale slepton masses to evolve to relatively high masses compared to the $F_{\Phi} \sim \mathbf{1}$ and $\mathbf{2 4}$ models, so that left sfermions are lighter than right sfermions; this is in contrast to usual expectations from models with universal gaugino masses. The $m_{H_{2}}^{2}$ mass parameter initially has an upward trajectory, but is ultimately evolved to negative values so that radiative electroweak symmetry is just barely broken.

A variety of physical sparticle masses along with the magnitude of the weak scale $\mu$ parameter are shown versus $\tan \beta$ in Fig. 3 for the four model choices using the same parameters as in Fig. 2. Frame $a$ ) shows the generic mSUGRA model spectrum for comparison with the models with non-universal gaugino masses. In frame $b$ ), the large mass gap between $m_{\widetilde{W}_{1}}$ or $m_{\widetilde{Z}_{2}}$ and $m_{\widetilde{Z}_{1}}$ is apparent. This mass gap has important consequences for collider experiments: frequently it is so large that neutralino decays to real $Z$ bosons are often allowed! Signatures involving real $Z_{\mathrm{s}}$ could be a distinctive signature for models leading to large mass gaps between $\widetilde{Z}_{2}$ and $\widetilde{Z}_{1}$.

For the $F_{\Phi} \sim 75$ case in frame $c$ ), there is almost no mass gap between $m_{\widetilde{W}_{1}}$ and $m_{\widetilde{Z}_{1}}$. For instance, for $\tan \beta=5$, with the other parameters as in the figure, $m_{\widetilde{W}_{1}}-m_{\widetilde{Z}_{1}}$ is just $0.5 \mathrm{GeV}$; this gap increases slightly with $\tan \beta$. The mass difference between $\widetilde{Z}_{2}$ and $\widetilde{Z}_{1}$ though larger $(\sim 18 \mathrm{GeV}$ for $\tan \beta=5)$ is still considerably smaller than in the canonical mSUGRA case. In this case, decays of $\widetilde{Z}_{2}$ and certainly $\widetilde{W}_{1}$ will lead to very soft visible particles which will make detection of hard isolated leptons from cascade decays very difficult. In view of the very tiny mass difference between the chargino and $\widetilde{Z}_{1}$, the reader may legitimately 
wonder whether the chargino is sufficently long lived as to travel a substantial distance in the detector, thus leaving a track before decaying. We have checked, however, 3 that the lifetime of the chargino is $\mathcal{O}\left(10^{-11} \mathrm{~s}\right)$ so that this appears not to be the case.

For the $F_{\Phi} \sim 200$ model in frame $d$ ), the $\widetilde{W}_{1}-\widetilde{Z}_{1}$ mass gap is just a few GeV, while $m_{\widetilde{Z}_{2}}-m_{\widetilde{Z}_{1}}$ is several tens of GeV. We have checked, however, that $\tau_{\widetilde{W}_{1}} \gtrsim 10^{-15}$ seconds, so that it decays rapidly without an appreciably displaced vertex. In this case, the $\widetilde{Z}_{4}$ is mainly a bino, and is the heaviest of all the sparticles.

Aside from that alteration of the masses, the weak scale values of the gaugino masses in Table [ I also imply very different mixing patterns for the charginos and neutralinos as compared to the usual mSUGRA case. In contrast to the mSUGRA case, $|\mu|$ tends to be somewhat smaller than $M_{2}$, and the lighter neutralinos and $\widetilde{W}_{1}$ are dominantly Higgsino-like in the $\mathbf{7 5}$ and $\mathbf{2 0 0}$ cases. This impacts on the decays of sparticles, e.g. $\widetilde{Z}_{2}$ and sometimes also $\widetilde{W}_{1}$ production in cascade decays tends to be suppressed, while frequently heavier charginos and neutralinos are produced with large rates. The decay patterns of $\widetilde{W}_{1}$ and $\widetilde{Z}_{2}$ are also changed from usual mSUGRA expectation. This will reflect itself in changes in expected rates for various event toplogies as we will see later.

The different boundary condition for gaugino masses sometimes has a strong effect on other masses via the RGE. For instance, for the $F_{\Phi} \sim \mathbf{2 0 0}$ model shown in frame $d$ ), the huge GUT scale value of $M_{1}=1250 \mathrm{GeV}$ causes right slepton and squark masses to evolve to large values so that in this case $m_{\tilde{e}_{R}}>m_{\tilde{q}}>m_{\tilde{e}_{L}}$ ! Another significant difference from the usual mSUGRA case is the large splittings between the masses of various squarks in the $\mathbf{7 5}$ and 200 cases. Indeed it is sometimes possible to have $m_{\tilde{q}_{L}} \geq m_{\tilde{g}} \geq m_{\tilde{q}_{R}}$, so that gluinos decay almost exclusively to right handed squarks. This, in turn, alters the cascade decay patterns from usual expectation because the right handed squarks cannot decay into charginos and neutralinos with dominant $S U(2)$ components.

In Fig. 1 , we show gluino and squark mass contours in the $m_{0} v s . M_{3}^{0}$ plane for $\tan \beta=5$, $A_{0}=0$ and $\mu>0$. The bricked regions are excluded by theoretical constraints: either electroweak symmetry is not broken appropriately, or the lightest SUSY particle (LSP) is not the lightest neutralino, in contradiction with results from searches for stable cosmological relics. These regions are sensitive to the exact choice of $m_{t}$. The gray shaded regions are excluded by collider search experiments for SUSY particles, and are mainly formed from the LEP2 bound that $m_{\widetilde{W}_{1}}>85.5 \mathrm{GeV}$ [20]; the LEP2 bound from the non-observation of $h$ plays a smaller role since for $\tan \beta=5, m_{h}$ is usually not small. The chargino bounds used may actually be too stringent for the $F_{\Phi} \sim 75$ and 200 models where the $m_{\widetilde{W}_{1}}-m_{\widetilde{Z}_{1}}$

\footnotetext{
${ }^{3}$ Because the mass gap is smaller than $1 \mathrm{GeV}$, it is not reasonable to compute the hadronic decay width of the chargino using ISAJET, which really computes the decay $\widetilde{W}_{1} \rightarrow q \bar{q} \widetilde{Z}_{1}$. Instead it is more reasonable to compute exclusive decays into 1,2 , etc. pion states in association with $\widetilde{Z}_{1}$. We are grateful to M. Drees who has provided us a code to do so. For the $\tan \beta=5$ point discussed in the text, the lifetime using this code agrees with the ISAJET lifetime to within a factor 2. For the decay of $\widetilde{Z}_{2}$ for which the mass gap is $\sim 20 \mathrm{GeV}$, the decays can, of course, be calculated using ISAJET.
} 
mass gap is small; for these cases, the LEP2 limits will have to be re-analyzed. I The gluino and squark mass contours are intended for comparison with the parameter space reach plots that will be presented in Sec. 3 of this paper. In Fig. 5, we show the same mass contours for $\tan \beta=25$. In this case, the parameter space is much more restrictive. In particular, for the $F_{\Phi} \sim 75$ model in frame $c$ ), radiative electroweak symmetry breaking is difficult to achieve for large values of the parameter $M_{3}^{0}$.

\section{EVENT SIMULATION AND REACH CALCULATIONS}

In several previous studies 9 9 13], a variety of signal channels for the discovery of mSUGRA (with universal GUT scale gaugino masses) at the Tevatron were investigated, and the reach of the Tevatron Main Injector era (MI-integrated luminosity of $2 \mathrm{fb}^{-1}$ ) and TeV33 era (integrated luminosity of $\sim 25 \mathrm{fb}^{-1}$ ) were delineated in the parameter space of the mSUGRA model. We had investigated [9,13] several promising discovery channels that included

- multi-jet $+E_{T}$ events (veto hard, isolated leptons) (J0L),

- events with a single isolated lepton plus jets $+\not_{T}(\mathrm{~J} 1 \mathrm{~L})$,

- events with two opposite sign isolated leptons plus jets $+\not_{T}(\mathrm{JOS})$,

- events with two same sign isolated leptons plus jets $+\mathbb{E}_{T}(\mathrm{JSS})$,

- events with three isolated leptons plus jets $+\mathbb{E}_{T}(\mathrm{~J} 3 \mathrm{~L})$,

- events with two isolated leptons $+E_{T}$ (no jets, clean) (COS),

- events with three isolated leptons $+E_{T}$ (no jets, clean) $(\mathrm{C} 3 \mathrm{~L})$.

In these samples, the number of leptons is exactly that indicated, so that these samples are non-overlapping. For Tevatron data samples on the order of $0.1 \mathrm{fb}^{-1}$, the J0L signal generally gave the best reach for supersymmetry. It is the classic signature for detecting

\footnotetext{
${ }^{4}$ Since the two lighter neutralinos contain significant Higgsino components, and $m_{\widetilde{Z}_{2}}-m_{\widetilde{Z}_{1}}$ is at least a few $\mathrm{GeV}$ for the $\mathbf{7 5}$ model (tens of $\mathrm{GeV}$ for the $\mathbf{2 0 0}$ model), we may expect LEP experiments might be able to detect signals from $e^{+} e^{-} \rightarrow \widetilde{Z}_{1} \widetilde{Z}_{2}$ production. For the $\mathbf{2 0 0}$ case, the non-observation of acollinear leptons or jets from $\widetilde{Z}_{2}$ decay could lead to significant limits on its mass. In the $\mathbf{7 5}$ case the analysis will have to be redone since $m_{\widetilde{Z}_{2}}-m_{\widetilde{Z}_{1}}$ is just a few $\mathrm{GeV}$, but it is worth keeping in mind that in the MSSM, ALEPH finds a mass bound of $79 \mathrm{GeV}$ on $m_{\widetilde{W}_{1}}$ that is derived by combining chargino and neutralino searches, assuming a mass gap $\geq 5 \mathrm{GeV}$. Finally, we note that in the $\mathbf{7 5}$ scenario, the branching fraction for the decay $\widetilde{Z}_{2} \rightarrow \widetilde{W}_{1} \ell \nu$ is significant; since the daughters of $\widetilde{W}_{1}$ are likely to be soft, $\widetilde{Z}_{1} \widetilde{Z}_{2}$ production could result in "monolepton" events at LEP. While it is clear that a dedicated analysis is required to really exclude the "hatched region" for the 200, and especially the $\mathbf{7 5}$ cases, we have chosen to show it using the same criteria in all four cases.
} 
gluinos and squarks at hadron colliders. For larger data samples typical of those expected at the MI or TeV33, the C3L signal usually yielded the greatest reach except when leptonic decays of charginos and neutralinos are strongly suppressed. In the present paper, we will extend these results for models with non-universal GUT scale gaugino masses.

We have found that the second model described above with $F_{\Phi} \sim \mathbf{2 4}$ can give rise to SUSY events at the Tevatron which are rich in $Z$ bosons. To extract this signal, we require:

- identification of a leptonic " $Z$ " boson $\left(Z \rightarrow e^{+} e^{-}\right.$or $\left.\mu^{+} \mu^{-}\right)$plus jets plus $\not_{T}(\mathrm{JZ})$.

To model the experimental conditions at the Tevatron, we use the toy calorimeter simulation package ISAPLT. We simulate calorimetry covering $-4<\eta<4$ with cell size $\Delta \eta \times \Delta \phi=0.1 \times 0.0872$. We take the hadronic (electromagnetic) energy resolution to be $70 \% / \sqrt{E}(15 \% / \sqrt{E})$. Jets are defined as hadronic clusters with $E_{T}>15 \mathrm{GeV}$ within a cone with $\Delta R=\sqrt{\Delta \eta^{2}+\Delta \phi^{2}}=0.7$. We require that $\left|\eta_{j}\right| \leq 3.5$. Muons and electrons are classified as isolated if they have $p_{T}>5 \mathrm{GeV},|\eta(\ell)|<2.5$, and the visible activity within a cone of $R=0.3$ about the lepton direction is less than $\max \left(E_{T}(\ell) / 4,2 \mathrm{GeV}\right)$. In our analysis, we neglect multiple scattering effects, non-physics backgrounds from photon or jet misidentification, and make no attempt to explicitly simulate any particular detector.

We incorporate in our analysis the following trigger conditions:

1. one isolated lepton with $p_{T}(\ell)>15 \mathrm{GeV}$ and $\not_{T}>15 \mathrm{GeV}$,

2. $E_{T}>35 \mathrm{GeV}$,

3. two isolated leptons each with $E_{T}>10 \mathrm{GeV}$ and $E_{T}>10 \mathrm{GeV}$,

4. one isolated lepton with $E_{T}>10 \mathrm{GeV}$ plus at least one jet plus $\not_{T}>15 \mathrm{GeV}$,

5. at least four jets per event, each with $E_{T}>15 \mathrm{GeV}$.

Thus, every signal or background event must satisfy at least one of the above conditions. In addition to these basic selection and trigger criteria, we impose various additional cuts listed in Ref. [9] the various signal classes. In particular, for the jetty channels, we require $E_{T}\left(j_{1}\right), E_{T}\left(j_{2}\right)$ and $E_{T}$ all to exceed a cut parameter $E_{T}^{c}$ which is chosen to maximize the reach, while for the clean trilepton (C3L) channel, we require rather hard leptons with $E_{T}\left(\ell_{1}, \ell_{2}, \ell_{3}\right) \geq(20,15,10) \mathrm{GeV}$.

We have generated the following physics background processes using ISAJET: $t \bar{t}$ production, $W+$ jets, $Z+$ jets, $W W, W Z$ and $Z Z$ production and QCD (mainly from $b \bar{b}$ and $c \bar{c}$ production). Each background subprocess was generated with the hard scattering subprocess final state particles in $p_{T}$ bins of $25-50 \mathrm{GeV}, 50-100 \mathrm{GeV}, 100-200 \mathrm{GeV}, 200-400$ $\mathrm{GeV}$ and 400-600 GeV. The numerical background values we use are listed in Ref. [9], and will not be repeated here.

For the new JZ event channel, we require two opposite sign same flavor isolated leptons $\left(e\right.$ or $\mu$ ) with $m(\ell \bar{\ell})$ within $M_{Z} \pm 8 \mathrm{GeV}$. We also require $n($ jets $) \geq 2, S_{T} \geq 0.2$ and $E_{T} \geq 40$ $\mathrm{GeV}$. In this case, the background rate was found to be $13.6 \mathrm{fb}$, mostly coming from $t \bar{t}, W Z$ and $Z Z$ production. 


\section{TEVATRON REACH RESULTS FOR SUGRA MODELS WITH NON-UNIVERSAL GAUGINO MASSES}

In Fig. 6-Fig. 21, we show the results of our computation of the SUSY reach of Tevatron collider experiments for models with non-universal gaugino masses. For each set of model input parameters, and for a given integrated luminosity, we consider a signal to be observable above background if, (for some value of the cut parameter $E_{T}^{c}$ for the jetty channels other than the $J Z$ channel)

- $S>5 \sqrt{B}$,

- $S>0.2 B$, and

- $S>5$ (10) for integrated luminosity equal to 0.1 or $2 \mathrm{fb}^{-1}\left(25 \mathrm{fb}^{-1}\right)$,

where $S$ is the expected number of signal events and $B$ is the expected number of background events. Within our framework, the scale of sparticle masses (and hence their production rates) is mainly determined by the parameters $m_{0}$ and $M_{3}^{0}$ (which fixes other gaugino masses at the unification scale). For this reason, the $m_{0}-M_{3}^{0}$ plane provides a convenient way to present our results. The results are somewhat less sensitive to variation of other parameters. In our analysis, we fix $A_{0}=0$ and choose $\mu>0$ (negative values of $\mu$ frequently do not yield the correct symmetry breaking pattern), and illustrate our results for $\tan \beta=5$ and 25 . Sampled points for which there is an observable signal for integrated luminosity of $0.1 \mathrm{fb}^{-1}$ are denoted by black squares; gray squares denotes points where the signal is observable with $2 \mathrm{fb}^{-1}$ and white squares, points that can be accessed with $25 \mathrm{fb}^{-1}$. Sampled points not accessible with even $25 \mathrm{fb}^{-1}$ of integrated luminosity are denoted with an $\times$.

\section{A. Reach via the J0L channel}

Fig. 6 shows results in the $m_{0}$ vs. $M_{3}^{0}$ plane for $\tan \beta=5, A_{0}=0$ and $\mu>0$. These results are in the J0L channel, which is the classic signature for supersymmetry at hadron colliders. For the $F_{\Phi} \sim \mathbf{1}$ case with universal gaugino masses in frame $a$ ), we find no reach for mSUGRA (black squares) with the current Tevatron data sample beyond the region already excluded at LEP2. However, experiments at the MI should be able to probe $M_{3}^{0}$ values up to $150 \mathrm{GeV}\left(m_{\tilde{g}} \simeq 400 \mathrm{GeV}\right)$ for lower values of $m_{0}$. The TeV33 integrated luminosity extends this reach to $M_{3}^{0}=175 \mathrm{GeV}$, corresponding to $m_{\tilde{g}} \simeq 480 \mathrm{GeV}$. For the $F_{\Phi} \sim 24$ model in frame $b$ ), there is a significant reach of current Tevatron experiments beyond the reach of LEP2. This is due mainly to the increased values of $m_{\widetilde{W}_{1}}$ and $m_{\widetilde{Z}_{2}}$ relative to their mSUGRA counterparts for a given value of $M_{3}^{0}$, so that just beyond the LEP2 limit, relatively light values of $m_{\tilde{g}} \sim 300 \mathrm{GeV}$ are still allowed, and can give rise to large J0L signals. The overall reach for SUSY in frame $b$ ) extends to $M_{3}^{0}=175 \mathrm{GeV}$, which is comparable to the mSUGRA case in frame $a$ ). For these large values of $M_{3}^{0}$, gluinos and squarks are heavy, and chargino and neutralino production is the dominant SUSY mechanism. For $M_{3}^{0}=175 \mathrm{GeV}, m_{\widetilde{W}_{1}}$ is significantly heavier in the $\mathbf{2 4}$ model relative to the mSUGRA case: the accessibility of heavier charginos is presumably due to the larger mass gap between the chargino and the LSP, which should increase the efficiency for detecting J0L events. For the $F_{\Phi} \sim 75$ model 
in frame $c$ ), the limits from LEP2 are again suppressed compared to the mSUGRA case due to heavier values of $m_{\widetilde{W}_{1}}$ for a given value of $M_{3}^{0}$. In this model, $m_{\widetilde{W}_{1}} \simeq m_{\widetilde{Z}_{1}}$ so that there is very little visible energy from $\widetilde{W}_{1}$ decays, and they behave effectively like the $\widetilde{Z}_{1}$ in the detector, i.e. they give missing energy. Gluino and squark pair production gives rise to a significant J0L signal for low values of $M_{3}^{0}$, so that there is still a substantial reach for SUSY via the MI and TeV33. The reach of TeV33 is somewhat smaller than in the mSUGRA case because for values of $M_{3}^{0} \simeq 175 \mathrm{GeV}$, direct $\widetilde{W}_{1}$ and $\widetilde{Z}_{2}$ production dominates $\tilde{g} \tilde{g}$ and $\tilde{g} \tilde{q}$ production: e.g. for mSUGRA, $\widetilde{W}_{1} \overline{\widetilde{W}}_{1}$ production leads to jets $+\mathbb{E}_{T}$ events, but for the $F_{\Phi} \sim 75$ model no hard jets get produced in $\widetilde{W}_{1}$ decay. Finally, the reach for the $F_{\Phi} \sim 200$ model is shown in frame $d$ ). In this model, as in the $\mathbf{7 5}$ case, relatively light values of $m_{\tilde{g}}$ are accessible to Tevatron experiments, and there is a significant reach for SUSY via the J0L signal. The black squares in the lower left of the frame come mainly from $\tilde{t}_{1} \tilde{t}_{1}$ and $\tilde{g} \tilde{g}$ events where $\tilde{g} \rightarrow b \tilde{b}_{1}$, so that the events are rich in $b$-jets. The ultimate reach of TeV33 again extends to $M_{3}^{0}=150 \mathrm{GeV}$ for low $m_{0}$, for which $m_{\tilde{g}} \simeq 400 \mathrm{GeV}$.

Similar results for the reach of the Tevatron via the J0L channel are shown in Fig. 7 for $\tan \beta=25$ (all other parameters are the same). The reach is somewhat diminished from the lower $\tan \beta$ case for all four models. Nevertheless, we see that there is significant reach via the Tevatron upgrades for supersymmetry in all models via the classic J0L channel.

\section{B. Reach via the J1L channel}

In Fig. 8 we show the Tevatron reach via the J1L signal for $\tan \beta=5$. For the mSUGRA case in frame $a$ ), there is no reach via the MI beyond the bounds from LEP2, but the TeV33 upgrade can access $M_{3}^{0}$ values as high as $\sim 175 \mathrm{GeV}$ for some parameter space points. For the $F_{\Phi} \sim \mathbf{2 4}$ model in frame $b$ ), the Tevatron MI has considerable reach for SUSY via the J1L channel beyond the LEP2 bounds. Much of the reach at lower $M_{3}^{0}$ values comes from gluino and squark cascade decays to $\widetilde{W}_{1}$ which then decays leptonically. The large mass gap between $\widetilde{W}_{1}$ and $\widetilde{Z}_{1}$ (shown in Fig. 3) results in a very energetic lepton which has a high probably for detection. TeV33 can access points with $M_{3}^{0} \simeq 175-200 \mathrm{GeV}$, corresponding to $m_{\tilde{g}} \sim 500 \mathrm{GeV}$. When we next examine the reach in the $F_{\Phi} \sim 75$ and $F_{\Phi} \sim 200$ models in frames $c$ ) and $d$ ), we see no reach via the MI, and only a marginal reach via TeV33. Much of the signal presumably comes from cascade decays to $\widetilde{Z}_{3}$ for which the branching fraction is substantial - the $\widetilde{Z}_{3}$ can then decay into real vector bosons to give the leptonic signal. In these cases, the small mass gap between $\widetilde{W}_{1}$ and $\widetilde{Z}_{1}$ yields low energy leptons with a poor probability to pass cuts in the J1L channel, and furthermore, cascade decays to these states tend to be somewhat suppressed.

For the $\tan \beta=25$ case in Fig. 9, in almost all the models, the reach via the J1L signal is diminished with respect to the lower $\tan \beta$ cases. Again, this is generally because at high $\tan \beta$, decays to $b$ 's and $\tau$ 's are enhanced relative to decays into $e$ 's and $\mu$ 's, making SUSY detection via leptonic modes in general more difficult. The exception here occurs with the $F_{\Phi} \sim 200$ model, where there is some reach for the MI beyond the LEP2 bounds. In this

case, some of the J1L events come from cascade decays involving $\widetilde{Z}_{3}$ which can decay via $\widetilde{Z}_{3} \rightarrow \widetilde{W}_{1} W$, and a hard lepton results from the $W$ decay. 


\section{Reach via the JOS channel}

The Tevatron reach via the JOS channel is illustrated in Fig. 10 and 11. For the mSUGRA case in frame a) of Fig. 10, there is some reach by the MI and TeV33 for low values of $m_{0}$ where sleptons become relatively light, and charginos and neutralinos can directly decay to sleptons and sneutrinos. The isolated dileptons come from a variety of cascade decay mechanisms involving charginos, neutralinos, sleptons and sneutrinos. For the $F_{\Phi} \sim 24$ model in frame $b$ ), there is a significant reach by Tevatron experiments beyond the LEP2 bounds even with the current data sample, and the reach expands considerably for the MI and TeV33. The OS dileptons again come from a variety of cascade decay mechanisms which include contributions from heavier charginos and neutralinos $\widetilde{W}_{2}$ and $\widetilde{Z}_{3}$. In the $F_{\Phi} \sim 75$ and $\mathbf{2 0 0}$ models, there is no reach beyond the LEP2 bounds for any Tevatron luminosity upgrade in this channel. This is, perhaps, not surprising if indeed the decays $\widetilde{Z}_{3} \rightarrow W \widetilde{W}_{1}$ are the main source of J1L events, since the $\widetilde{W}_{1}$ is mostly invisible; i.e. any JOS event is doubly suppressed by the branching fraction of the cascade decay of gluino or squark into a lepton. We see a similar pattern for the $\tan \beta=25$ case shown in Fig. 11, except also that the reach in the mSUGRA and $F_{\Phi} \sim \mathbf{2 4}$ models is diminished due to the enhancement of decays to $\tau$-leptons and $b$-quarks.

\section{Reach via the JSS channel}

The reach for SUSY in the JSS channel has been noted as a distinct signal for cascade decays of the $\tilde{g}$ to $\widetilde{W}_{1}$, where the Majorana nature of the gluino gives rise to equal probability for detection of same-sign and opposite-sign dileptons. In the mSUGRA model in Fig. 12a), there is only a tiny region that can be probed at the Tevatron in this channel mainly because the LEP2 bounds force $m_{\tilde{g}}$ and $m_{\tilde{q}}$ to such high values that their production cross section is suppressed relative to direct chargino, neutralino and slepton pair production. For the $F_{\Phi} \sim \mathbf{2 4}$ model, however, lighter values of $m_{\tilde{g}}$ are allowed beyond the LEP2 exclusion region, and furthermore, the large $\widetilde{W}_{1}-\widetilde{Z}_{1}$ decay gap gives rise to a relatively high probability to detect $\tilde{g} \rightarrow \widetilde{W}_{1} \rightarrow \ell$ cascade decay leptons. Consequently, we see a significant reach in the JSS channel in frame $b$ ). Nonetheless, the reach is somewhat smaller than in the JOS channel, which also receives significant contributions from leptonic decays of neutralinos. In the $F_{\Phi} \sim 75$ and 200 models, there appears to be no signal in the JSS channel beyond the LEP2 region for much the same reasons that we just discussed for the JOS case. Broadly similar results hold for the $\tan \beta=25$ case illustrated in Fig. 13, where we see the usual reduction in the region where there is an observable signal in the mSUGRA and in the $F_{\Phi} \sim 24$ models.

\section{E. Reach via the J3L channel}

In Fig. 14, we show the reach of Tevatron experiments in the J3L channel. There is a significant reach by the Tevatron MI and TeV33 for mSUGRA for $m_{0} \lesssim 150 \mathrm{GeV}$ extending all the way to $M_{3}^{0}=225 \mathrm{GeV}$, corresponding to $m_{\tilde{g}} \simeq 600 \mathrm{GeV}$, as shown in frame $a$ ). The mSUGRA J3L signal dominantly comes from direct chargino, neutralino, slepton and 
sneutrino production and decays. In the $F_{\Phi} \sim \mathbf{2 4}$ model, there is a significant reach in the J3L channel even for large values of $m_{0}$ since $m_{\tilde{g}}$ can be as light as $\simeq 230 \mathrm{GeV}$ just beyond the LEP2 bound. For the largest values of $M_{3}^{0}$ where there is an observable signal in the mSUGRA and $\mathbf{2 4}$ cases, sparticle production is dominated by chargino and neutralino production, and the signal dominantly comes from $\widetilde{W}_{1} \widetilde{Z}_{2}$ production with jets coming from QCD radiation. Because we only require leptons to have $E_{T}(\ell) \geq 10 \mathrm{GeV}$, we expect that the efficiency increases by a relatively small amount despite the increase in $m_{\widetilde{W}_{1}}-m_{\widetilde{Z}_{1}}$ in going from the mSUGRA to the $\mathbf{2 4}$ case (in contrast to the case of the J0L signal where the increase in efficiency might be substantial). As a result the boundary of the TeV33 region occurs for similar values of $m_{\widetilde{W}_{1}} \sim 170-180 \mathrm{GeV}$. For the $F_{\Phi} \sim 75$ and 200 models, there is again hardly any reach for SUSY in the J3L channel. For the large $\tan \beta=25$ case illustrated in Fig. 15, the reach for all models in the J3L channel is diminished due to enhanced decays to 3rd generation particles, but for the $\mathbf{2 4}$ case, there is still a significant region beyond the current LEP reach that can be probed at Tevatron upgrades.

\section{F. Reach via the C3L channel}

The clean trilepton signal which often comes from $\widetilde{W}_{1} \widetilde{Z}_{2} \rightarrow 3 \ell+E_{T}$ has frequently been considered the most promising signal via which to search for SUSY at luminosity upgrades of the Tevatron. These analyses have mainly been performed within the mSUGRA model. But even in this case, it has been known for some time that there are parameter space regions where there is no observable signal in this channel because chargino and neutralino decays to leptons may be suppressed. Our computation of the reach in this C3L channel is shown in Fig. 16 for $\tan \beta=5$. For the mSUGRA model, the reach of the MI extends to $M_{3}^{0}=200 \mathrm{GeV}$, while the TeV33 reach extends past $M_{3}^{0}=250 \mathrm{GeV}$, corresponding to $m_{\tilde{g}} \simeq 650 \mathrm{GeV}$ ! The well known gap in the reach at $m_{0} \simeq 200-300 \mathrm{GeV}$ due to destructive interference in neutralino leptonic decays is clearly visible. There is substantial reach for SUSY in the $F_{\Phi} \sim \mathbf{2 4}$ model both at the MI and TeV33. The reach in $M_{3}^{0}$ at low $m_{0}$ is, however, diminished relative to the mSUGRA model. This is because the large mass gap between $m_{\widetilde{Z}_{2}}$ and $m_{\widetilde{Z}_{1}}$ allows the spoiler decay modes $\widetilde{Z}_{2} \rightarrow Z \widetilde{Z}_{1}$ and $\widetilde{Z}_{2} \rightarrow h \widetilde{Z}_{1}$ to turn on at lower values of $M_{3}^{0}$. It is, however, interesting to see that there is an observable signal beyond the LEP bounds, for all values of $m_{0}$ scanned in the figure. For the $F_{\Phi} \sim 75$ and 200 models, there is no reach for SUSY in the C3L channel, which underscores the model dependence of the much touted C3L signal. It may be of interest to examine whether the use of softer cuts [15] on the leptons affects this conclusion.

In the large $\tan \beta=25$ case of Fig. 17, the mSUGRA reach is diminished at low $m_{0}$ due to enhanced decays to $\tau$ leptons. It may be possible to further enhance this reach by softening the cuts on the leptons [15]. There remains a significant reach for SUSY in the C3L channel for the $F_{\Phi} \sim \mathbf{2 4}$ model at large $\tan \beta$ because the large mass gap between $m_{\widetilde{Z}_{2}}$

and $m_{\widetilde{Z}_{1}}$ allows $\widetilde{Z}_{2}$ decays to real selectrons and smuons to compete with decays to staus.

We have also checked the reach via the COS channel. While there are parameter regions where this could provide confirmation of a signal in other channels, the COS topology does not appear to increase the reach beyond what is observable via other channels. 


\section{G. Reach via the JZ channel}

It is possible to produce real $Z$ bosons in SUSY particle cascade decay events. Events with an identified $Z$ boson plus $E_{T}$ are interesting because Standard Model backgrounds to these mainly come from vector boson pair production or $t \bar{t}$ production where the leptons from the decays of the tops accidently reconstruct the $Z$ mass, and hence, are small. Prospects for observing just this signal at the Tevatron collider were examined long ago in the context of the MSSM framework [22]. In this study, the focus was on relatively small values of $\mu$ and $m_{\tilde{g}}$, so that all the charginos and neutralinos were accessible via the production and subsequent decays of gluinos.

Although JZ events are possible within the mSUGRA framework, they typically occur at very low rates, at least for sparticle masses accessible at the Tevatron. To understand this, we first recall that because $|\mu| \gg M_{1}, M_{2}$ as is typical in mSUGRA, the lighter neutralinos and the lighter chargino are mainly gaugino-like, while the heavier ones are higgsino-like. But since the $Z$ boson couples only to higgsino pairs or charged gaugino pairs, it is clear that

the widths for the decays $\widetilde{Z}_{3,4} \rightarrow \widetilde{Z}_{1,2} Z$ or $\widetilde{W}_{2} \rightarrow \widetilde{W}_{1} Z$ are suppressed by gaugino-Higgsino mixing angles.

Our results for the observability of SUSY events in the JZ channel are shown in Fig. 18 for $\tan \beta=5$, and in Fig. 19 for $\tan \beta=25$. Indeed we see from Fig. $18 a$ that there is no reach at either the Tevatron MI or TeV33 for mSUGRA in the JZ channel. However, for the $F_{\Phi} \sim \mathbf{2 4}$ model, in frame $\left.b\right)|\mu| \sim M_{2}$, and the $\widetilde{Z}_{3}$ can be light enough that it can be directly produced in collider events, while its decay branching fraction to $Z$ is substantial: $\sim 10-50 \%$. Also, the large $\widetilde{Z}_{2}-\widetilde{Z}_{1}$ mass gap allows the decay $\widetilde{Z}_{2} \rightarrow Z \widetilde{Z}_{1}$ to occur (via the subdominant higgsino component of $\widetilde{Z}_{1}$ ) in much of parameter space. We see in Fig. $18 b)$ that while this signal might be detectable at the MI for a limited range of parameters, the reach of $\mathrm{TeV} 33$ in this channel is indeed substantial, covering much of parameter space below $M_{3}^{0} \lesssim 150 \mathrm{GeV}$ ! Meanwhile, for the $F_{\Phi} \sim 75$ and 200 models, there is again no reach for SUSY in the JZ channel - the branching fractions for cascade decays to heavier neutralinos and charginos tend to be small in these cases.

For the $\tan \beta=25$ case in Fig. 19, there is again no reach for SUSY in the mSUGRA model or the $F_{\Phi} \sim 75$ and 200 models. In the $F_{\Phi} \sim 24$ model, there is a significant Tevatron reach in the JZ channel, but only for TeV33 type integrated luminosities. Since the JZ signal occurs at an observable level only in rather special models, the observation of such a signal in tandem with more conventional SUSY signals would be especially interesting since it could stringently restrict the underlying framework.

\section{SUMMARY AND CONCLUDING REMARKS}

The search for SUSY has become a standard item on all high energy physics experiments searching for physics beyond the Standard Model. For the most part, the analyses of current experiments as well as projections of capabilities of future experiments have have been carried out within the framework of the mSUGRA model, or within the MSSM framework with some ad hoc assumptions motivated by mSUGRA about scalar and gaugino masses. Since SUSY cross sections, after experimental cuts, are expected to be sensitive to sparticle mass and mixing patterns (which are determined by the presently unkown dynamics of SUSY 
breaking), it is worthwhile to examine just how much the SUSY reach of future facilities change in alternative scenarios.

These considerations motivated us to examine SUSY signals at Tevatron upgrades in the supergravity $S U(5)$ model [8] with non-universal gaugino masses at the GUT scale. For simplicity, other parameters were considered to unify as in the mSUGRA model. Such a scenario can be realized if there is a superfield $\Phi$ that is charged under $S U(5)$ and whose auxiliary component develops a vev that breaks the GUT gauge group down to the Standard Model gauge group. The resulting GUT scale gaugino masses are determined by the transformation properties of $\Phi$, which can transform as the $\mathbf{1}$ (this corresponds to mSUGRA), $\mathbf{2 4}, \mathbf{7 5}$ or the $\mathbf{2 0 0}$ dimensional representation of $S U(5)$. The resulting gaugino mass ratios at the GUT scale along with their renormalized values at the weak scale (relevant for phenomenology) are shown in Table I. The phenomenology is altered not only because of the differences in these weak scale gaugino masses, but also because the difference in the boundary condition on gaugino masses alters the renormalization group evolution of other parameters as well.

Our main result is the reach of Tevatron Main Injector and its possible TeV33 luminosity upgrade for the cases where $\Phi$ belongs to any one of these irreducible representations. We have examined this reach for various event topologies. The results of our calculation are shown in Figs. 6-19. The cumulative reach for SUSY, i.e. the region of the $m_{0}-M_{3}^{0}$ plane where there should be an observable signal in at least one of the channels, is shown in Figs 20 and 21. The precise reach is model-dependent. In Fig. 20 $a$, the reach in the mSUGRA model is built entirely out of the reach in the J0L and C3L channels. For some of the points examined, there may be observable signals in other channels as well. The reach of the Tevatron for the $F_{\Phi} \sim \mathbf{2 4}$ model is built out of the J0L, C3L and JZ channels, i.e. for a few points the SUSY signal appears to be observable only via the JZ channel, and not in the more standard J0L and C3L channels. In addition, over much of the observable parameter space, signals should also be detectable in many different leptonic channels. The additional signals should help in constraining the underlying model. In contrast, the reach in the $\mathbf{7 5}$ and $\mathbf{2 0 0}$ models shown in Fig. $20 c$ ) and $d$ ), the cumulative reach plot coincides with the reach plot for the J0L channel (Fig. 6)! In fact, the leptonic signals for SUSY will be observable for only extremely restricted regions of model parameters. This underscores the importance of the J0L channel in that it is relatively model independent, at least so long as the LSP is a stable neutralino which escapes detection: experimentalists should scrutinize this channel closely even if no leptonic SUSY signals can be seen. We should also mention that in our analysis, we have not attempted to really optimize the reach in this channel. By judiciously choosing the cuts, it may be possible to increase the reach somewhat beyond what appears in the figure.

For the $\tan \beta=25$ case shown in Fig. 21, the cumulative reach for mSUGRA shown in frame $a$ ) is again defined by just the J0L and C3L channels but is somewhat reduced relative to the corresponding low $\tan \beta$ case. The reach for the $F_{\Phi} \sim \mathbf{2 4}$ model shown in frame $b$ ) is again defined by the J0L, C3L and JZ channels, which underscores the importance of an independent search for SUSY in the JZ channel. The reach is only slightly diminished from the $\tan \beta=5$ case. The Tevatron SUSY reach for the $F_{\Phi} \sim \mathbf{7 5}$ and 200 models for $\tan \beta=25$ is again defined solely by the J0L channel; very few of these parameter space points are accessible in any other channel. Thus, a SUSY discovery with a signal only in 
the J0L channel may indicate non-universal gaugino masses which act to suppress leptonic signals originating from SUSY particle cascade decays.

In summary, we have examined the SUSY reach of luminosity upgrades of the Tevatron in non-minimal SUGRA type models where gaugino masses are not unified at some high scale. We find that rates for various signal topologies (and hence, the reach) can be quite different from mSUGRA expectations. There may be new signatures such as the high $p_{T}$

$Z+E_{T}$ signal in the $\mathbf{2 4}$ model that are unobservable in the mSUGRA picture. On the other hand, in the $\mathbf{7 5}$ and $\mathbf{2 0 0}$ models a signal might be observable only in the canonical multijet $+E_{T}$ channel. This is in contrast to $R$-parity violating models [23] where there might be observable signals only in the multilepton channel, but no signal in the usual $\mathbb{E}_{T}$ channel. We thus conclude that while it might well be possible to discover a signal for new physics at the Tevatron, its interpretation will have to be done with care. What we do not see, in addition of course to what we do see, may play an important role in unravelling the nature of the new physics.

\section{ACKNOWLEDGMENTS}

This research was supported in part by the U. S. Department of Energy under contract number DE-FG02-97ER41022, DE-FG-03-94ER40833 and DE-FG02-91-ER4086. 


\section{REFERENCES}

[1] A. Chamseddine, R. Arnowitt and P. Nath, Phys. Rev. Lett. 49, 970 (1982); R. Barbieri, S. Ferrara and C. Savoy, Phys. Lett. B119, 343 (1982); L.J. Hall, J. Lykken and S. Weinberg, Phys. Rev. D27, 2359 (1983); for a review, see H. P. Nilles, Phys. Rep. 110, 1 (1984).

[2] For recent reviews, see X. Tata, Lectures presented at the IX Jorge Swieca Summer School, Campos do Jordão, Brazil, Feb. 1997, UH-511-872-97, hep-ph/9706307; S. Dawson, Lectures at TASI 97, hep-ph/9712464 (1997).

[3] E. Cremmer, S. Ferrara, L. Girardello, and A. Van Proeyen, Phys. Lett. 116B, 231 (1982).

[4] C.T. Hill, Phys. Lett 135B, 47 (1984).

[5] Q. Shafi and C. Wetterich, Phys. Rev. Lett 52, 875 (1984).

[6] J. Ellis, K. Enqvist, D. Nanopoulos and K. Tamvakis, Phys. Lett. 155B, 381 (1985).

[7] M. Drees, Phys. Lett. 158B, 409 (1985).

[8] G. Anderson et al., in New Directions for High Energy Physics, Snowmass 96, ed. by D. G. Cassel, L. Trindle Gennari and R. H. Siemann; see also J. Amundson et al., in New Directions for High Energy Physics, Snowmass 96, ed. by D. G. Cassel, L. Trindle Gennari and R. H. Siemann.

[9] H. Baer, C-H. Chen, C. Kao and X. Tata, Phys. Rev. D52, 1565 (1995); H. Baer, C-H. Chen, F. Paige and X. Tata, Phys. Rev. D54, 5866 (1996).

[10] S. Mrenna, G. Kane, G. Kribs and J. Wells, Phys. Rev. D53, 1168 (1996).

[11] D. Amidei, R. Brock et al., Report of the TeV 2000 Working Group, Fermilab-Pub96/082 (1996).

[12] S. Mrenna et al., in Proc. 1996 Snowmass Summer Study.

[13] H. Baer, C-H. Chen, M. Drees, F. Paige and X. Tata, Phys. Rev. Lett. 79, 986 (1997) Phys. Rev. D58, 075008 (1998).

[14] H. Baer, K. Hagiwara and X. Tata, Phys. Rev. D35, 1598 (1987) R. Arnowitt and P. Nath, Mod. Phys. Lett. A2, 331 (1987); H. Baer and X. Tata, Phys. Rev. D47, 2739 (1993); H. Baer, C. Kao and X. Tata Phys. Rev. D48, 5175 (1993); S. Mrenna, G. Kane, G. D. Kribs and J. D. Wells, Phys. Rev. D53, 1168 (1996).

[15] V. Barger, C. Kao and T. Li, Phys. Lett. B433, 328 (1998); V. Barger and C. Kao, hep-ph/9811489 (1999); H. Baer, M. Drees, F. Paige, P. Quintana and X. Tata, FSUHEP-990222 (1999) (in preparation).

[16] F. Paige, S. Protopopescu, H. Baer and X. Tata, hep-ph/9804321 (1998).

[17] N. Arkani-Hamed, H-C. Cheng and T. Moroi, Phys. Lett. B387, 529 (1996).

[18] A. Brignole, L. Ibanez and C. Munoz, Nucl. Phys. B422, 125 (1994), Erratum, ibid., B436, 747 (1995); A. Brignole, L. Ibanez, C. Munoz and C. Scheich, Z. Phys. C74, 157 (1997).

[19] C. H. Chen. M. Drees and J. Gunion, Phys. Rev. Lett. 76, 2002 (1996) and Phys. Rev. D55, 330 (1997).

[20] R. Barate et al. (ALEPH Collaboration), Eur. Phys. J. C2, 417 (1998).

[21] M. Hohlmann, Fermilab-Conf-96/330-E (1996).

[22] H. Baer, X. Tata and J. Woodside, Phys. Rev. D42, 1450 (1990).

[23] H. Baer, C. Kao and X. Tata, Phys. Rev. D51, 2180 (1995). 


\section{TABLES}

\begin{tabular}{|c|ccr|ccc|}
\hline \hline \hline & & $M_{G U T}$ & & $M_{Z}$ & \\
$F_{\Phi}$ & $M_{3}$ & $M_{2}$ & $M_{1}$ & $M_{3}$ & $M_{2}$ & $M_{1}$ \\
\hline $\mathbf{1}$ & 1 & 1 & 1 & $\sim 6$ & $\sim 2$ & $\sim 1$ \\
$\mathbf{2 4}$ & 2 & -3 & -1 & $\sim 12$ & $\sim-6$ & $\sim-1$ \\
$\mathbf{7 5}$ & 1 & 3 & -5 & $\sim 6$ & $\sim 6$ & $\sim-5$ \\
$\mathbf{2 0 0}$ & 1 & 2 & 10 & $\sim 6$ & $\sim 4$ & $\sim 10$ \\
\hline \hline
\end{tabular}

TABLE I. Relative gaugino masses at $M_{G U T}$ and $M_{Z}$ in the four possible $F_{\Phi}$ irreducible representations. 


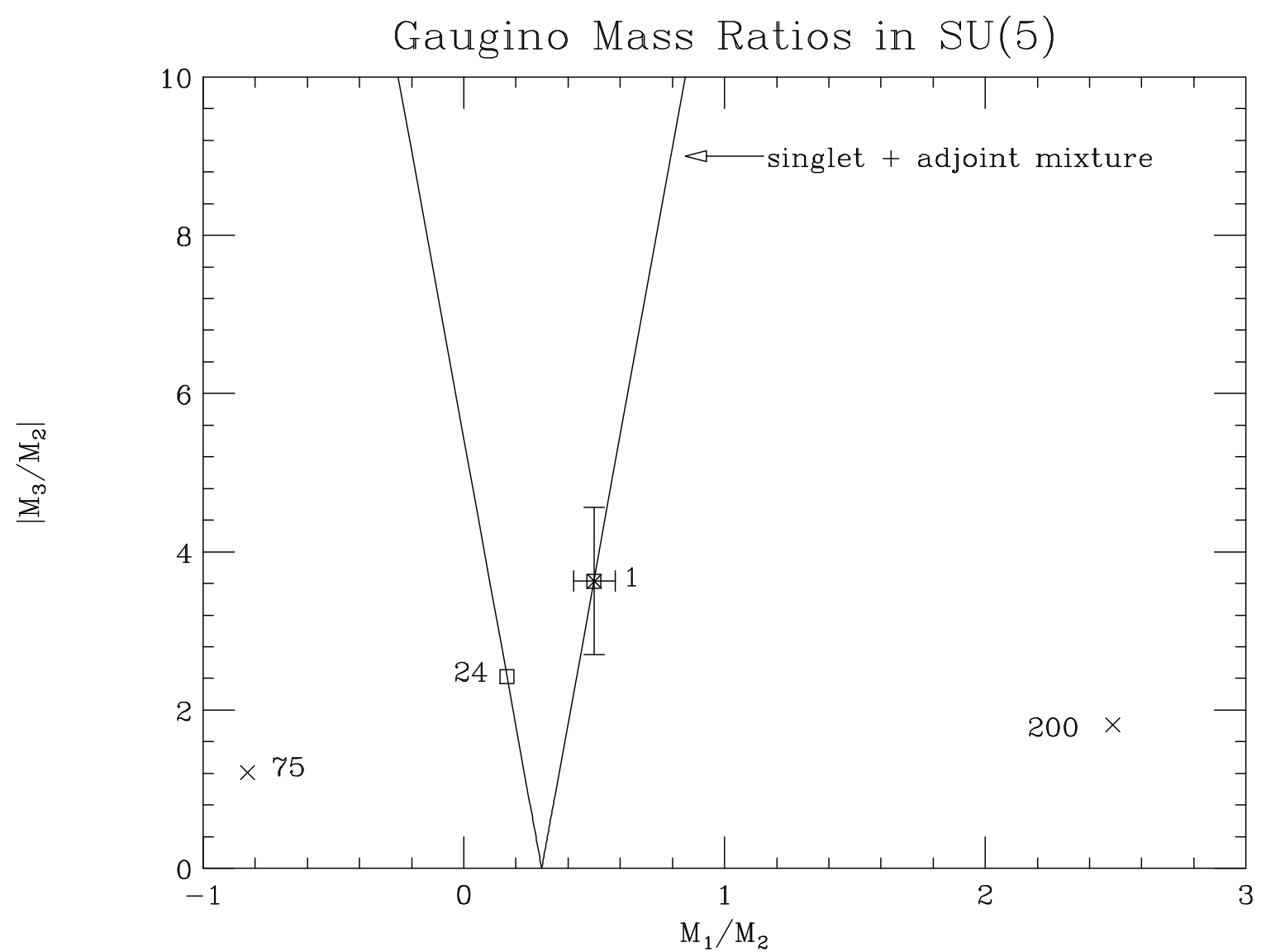

FIG. 1. Gaugino mass ratios for the four special cases where the field $\Phi$ transforms as the 1, 24, 75 or 200 dimensional representation of $S U(5)$, or as an arbitrary linear combination of the singlet and adjoint representations (solid line). 

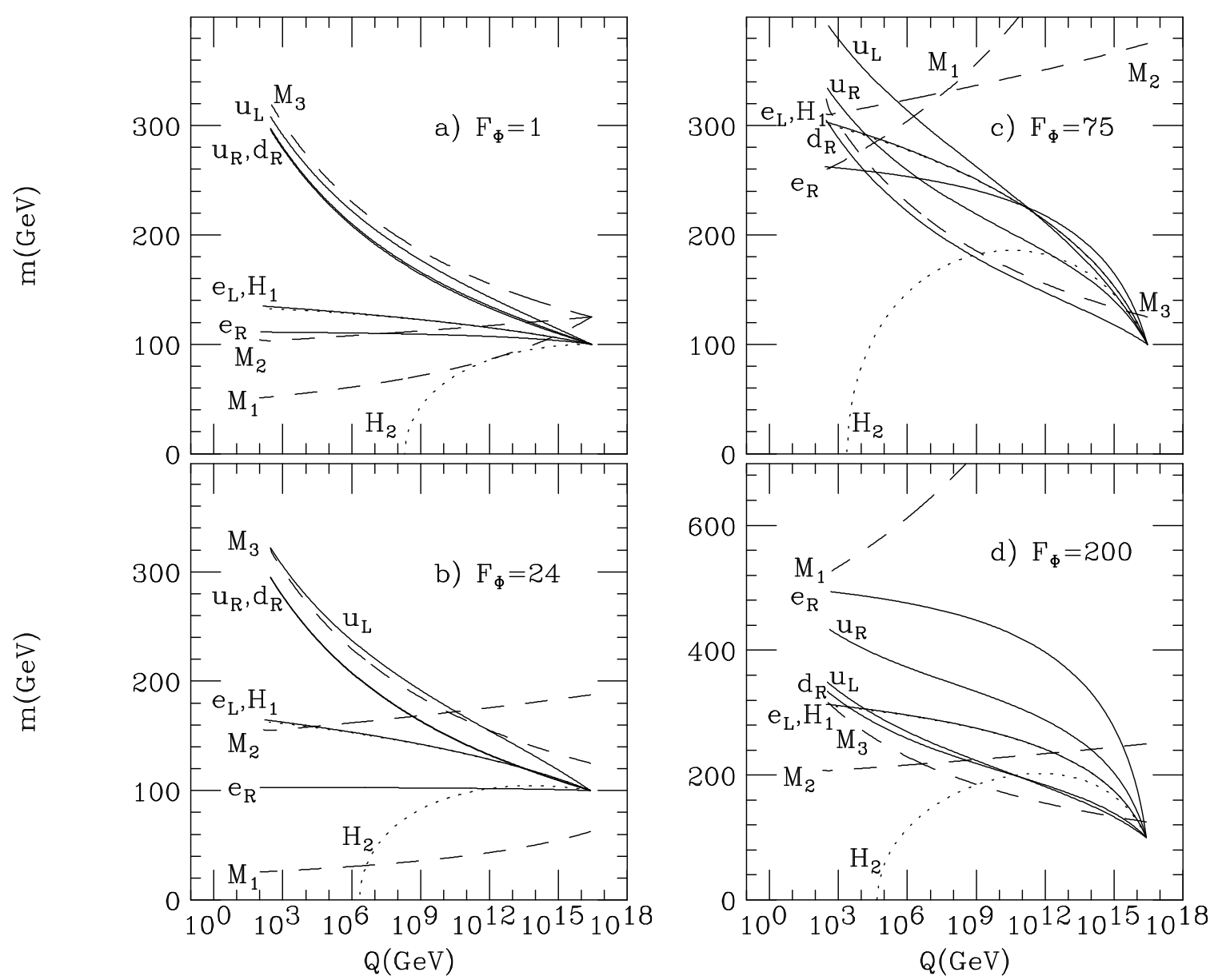

FIG. 2. A plot of the evolution of soft SUSY breaking parameters versus renormalization scale $Q$ from $M_{G U T}$ to $M_{\text {weak }}$ for SUGRA model parameters $m_{0}=100 \mathrm{GeV}, M_{3}^{0}=125 \mathrm{GeV}, A_{0}=0$, $\tan \beta=5$ and $\mu>0$, for the a) $\left.\left.F_{\Phi} \sim 1, b\right) F_{\Phi} \sim 24, c\right) F_{\Phi} \sim 75$ and $\left.d\right) F_{\Phi} \sim 200$ models. We take $m_{t}=175 \mathrm{GeV}$. Notice the different scale in frame $d$ ) 

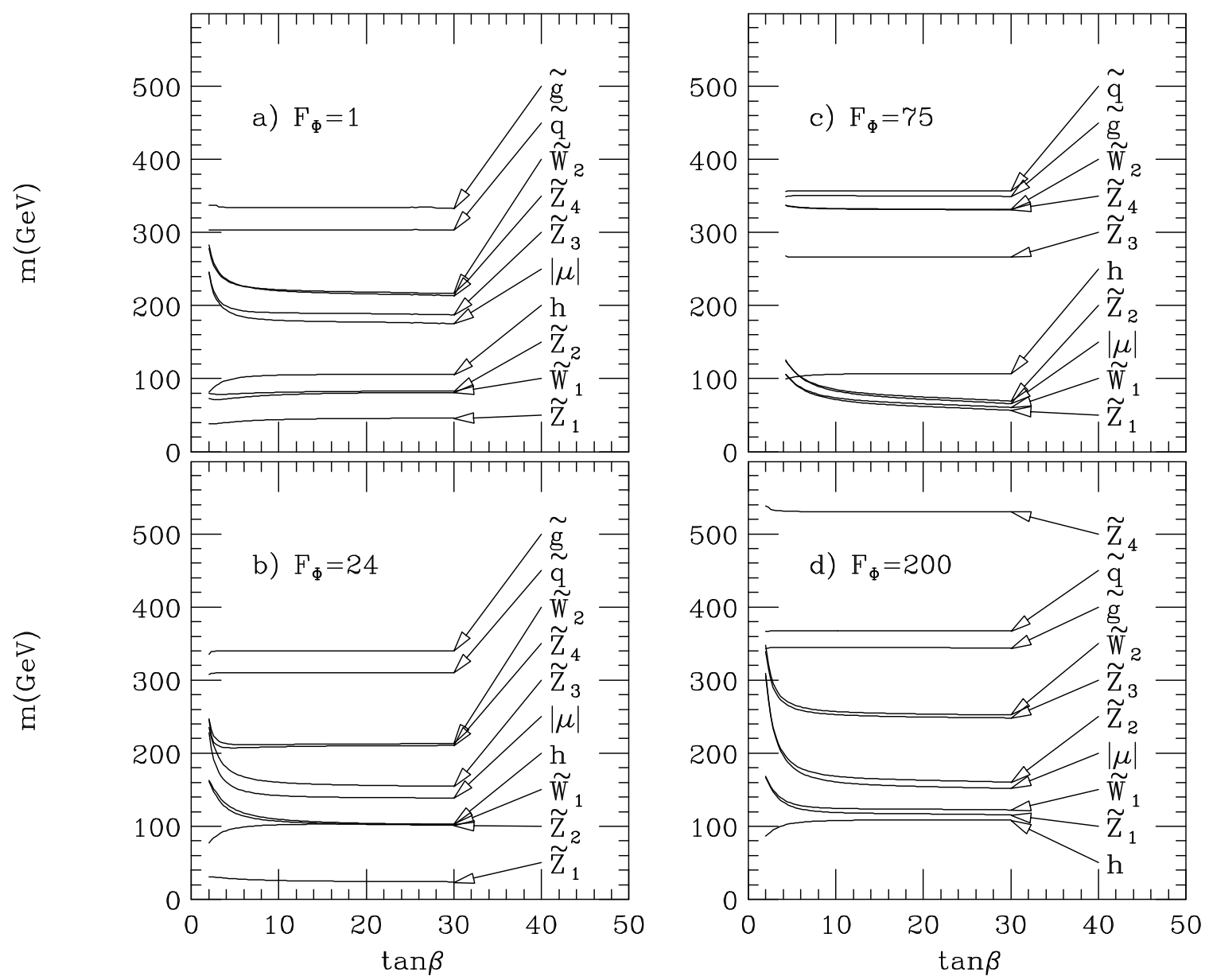

FIG. 3. A plot of various physical sparticle masses and the magnitude of the $\mu$ parameter versus $\tan \beta$ for SUGRA model parameters $m_{0}=100 \mathrm{GeV}, M_{3}^{0}=125 \mathrm{GeV}, A_{0}=0$ and $\mu>0$, for the a) $\left.\left.F_{\Phi} \sim 1, b\right) F_{\Phi} \sim 24, c\right) F_{\Phi} \sim 75$ and $d$ ) $F_{\Phi} \sim 200$ models. The squark mass is averaged over the first two generations. 

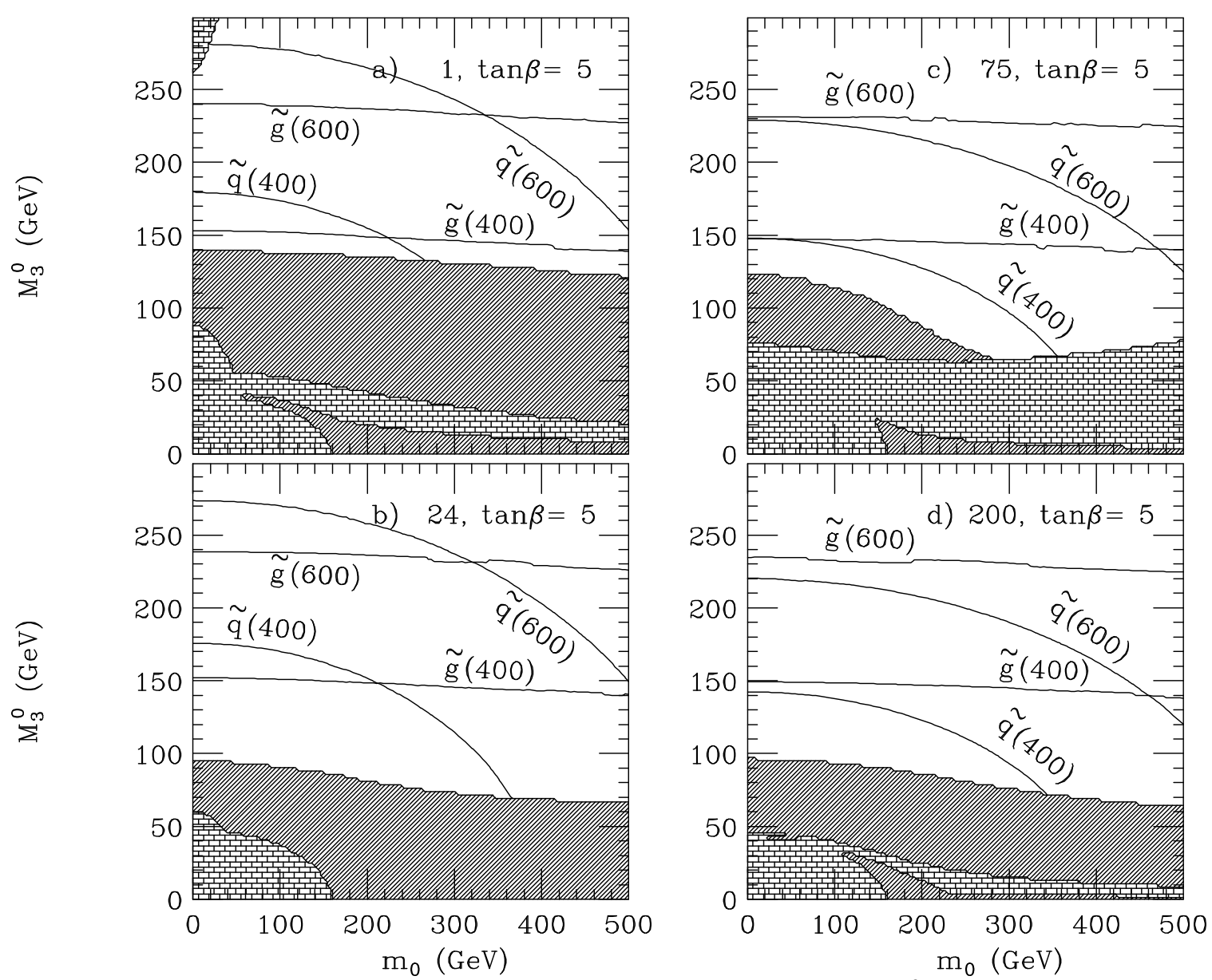

FIG. 4. A plot of gluino and squark mass contours in the $m_{0} v s . M_{3}^{0}$ plane for SUGRA model parameters $A_{0}=0, \tan \beta=5$ and $\mu>0$ for the a) $\left.\left.F_{\Phi} \sim 1, b\right) F_{\Phi} \sim 24, c\right) F_{\Phi} \sim 75$ and $d$ ) $F_{\Phi} \sim 200$ models. The squark mass is averaged over the first generation. 

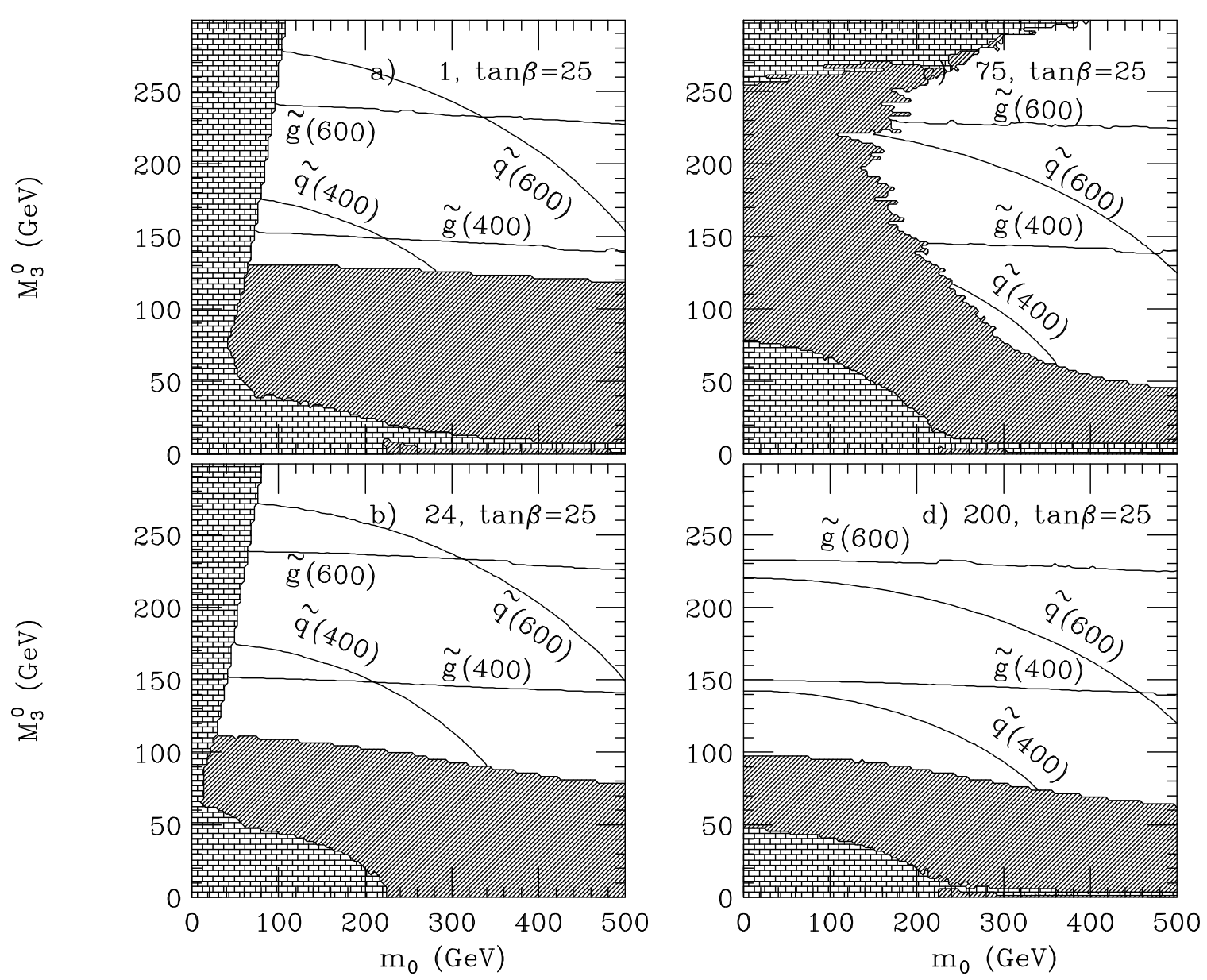

FIG. 5. Same as Fig. 4 except for $\tan \beta=25$. 

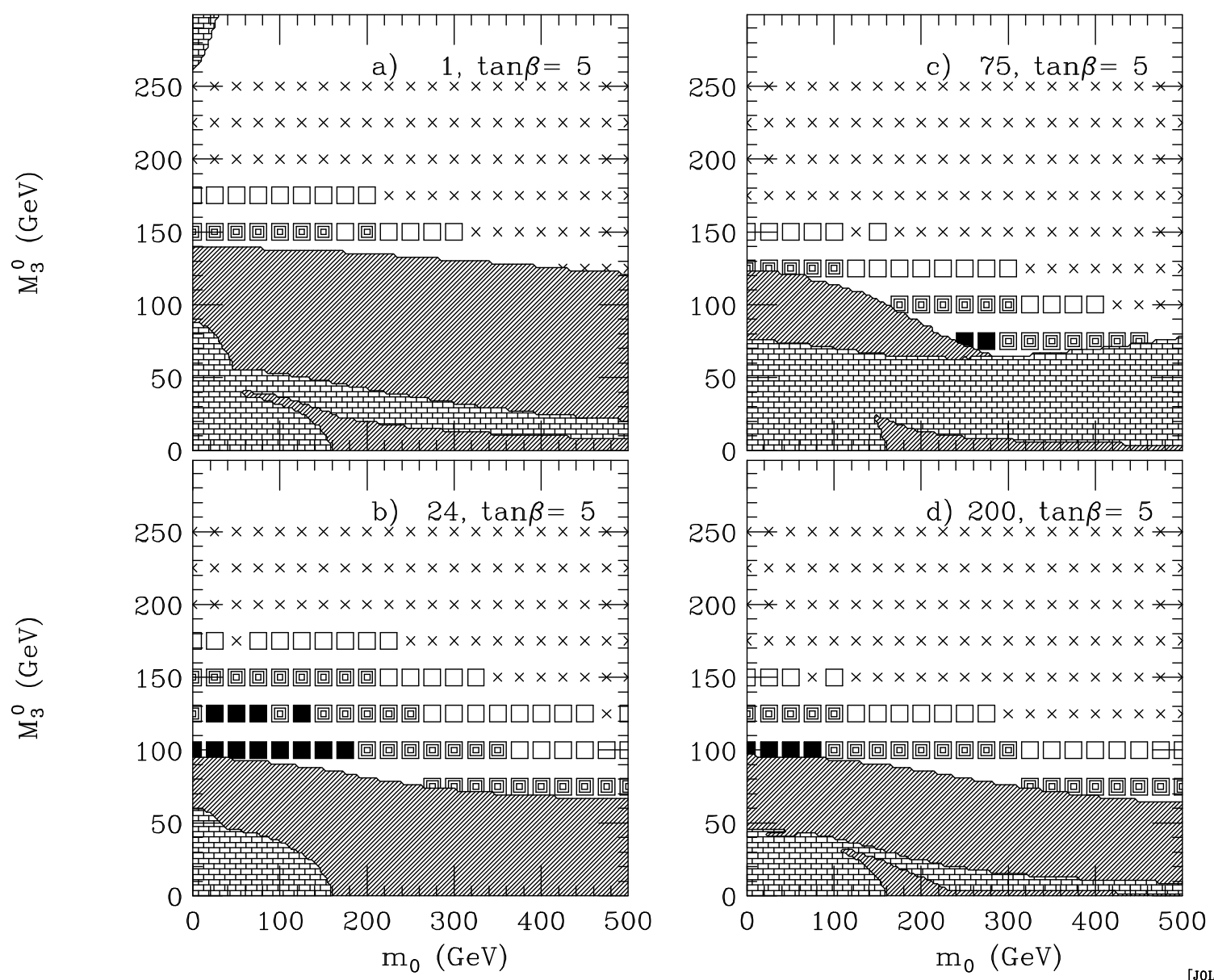

FIG. 6. A plot of parameter space points accessible to Fermilab Tevatron collider experiments with integrated luminosity $0.1 \mathrm{fb}^{-1}$ (black squares), $2 \mathrm{fb}^{-1}$ (gray squares) and $25 \mathrm{fb}^{-1}$ (white squares) via the multijet $+E_{T}$ signal (J0L). Events containing isolated leptons have been vetoed. Points are plotted in the $m_{0}$ vs. $M_{3}^{0}$ plane for SUGRA model parameters $A_{0}=0, \tan \beta=5$ and $\mu>0$ for the $\left.\left.a) F_{\Phi} \sim 1, b\right) F_{\Phi} \sim 24, c\right) F_{\Phi} \sim 75$ and $\left.d\right) F_{\Phi} \sim 200$ models. 

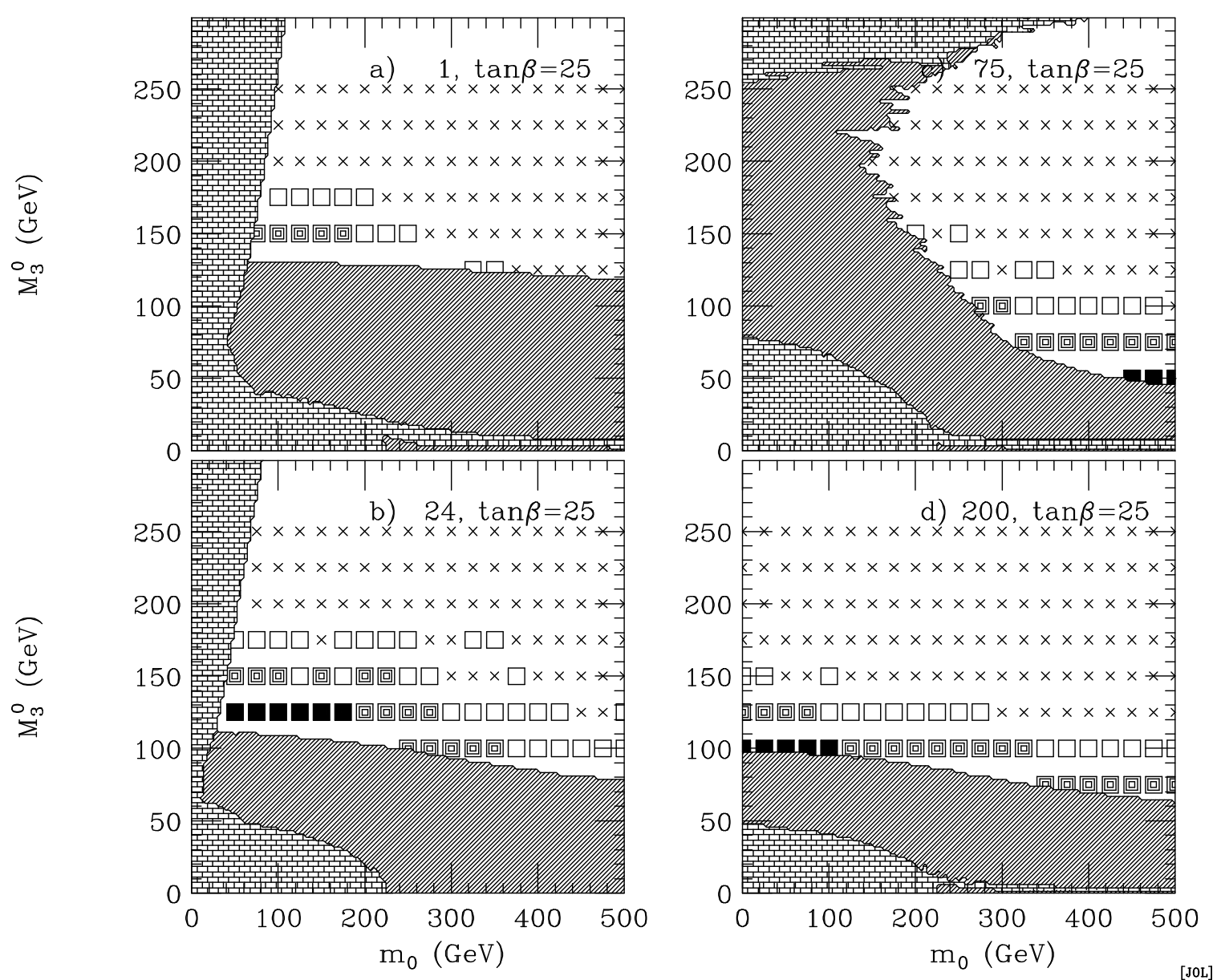

FIG. 7. The same as Fig. 6, except for $\tan \beta=25$. 

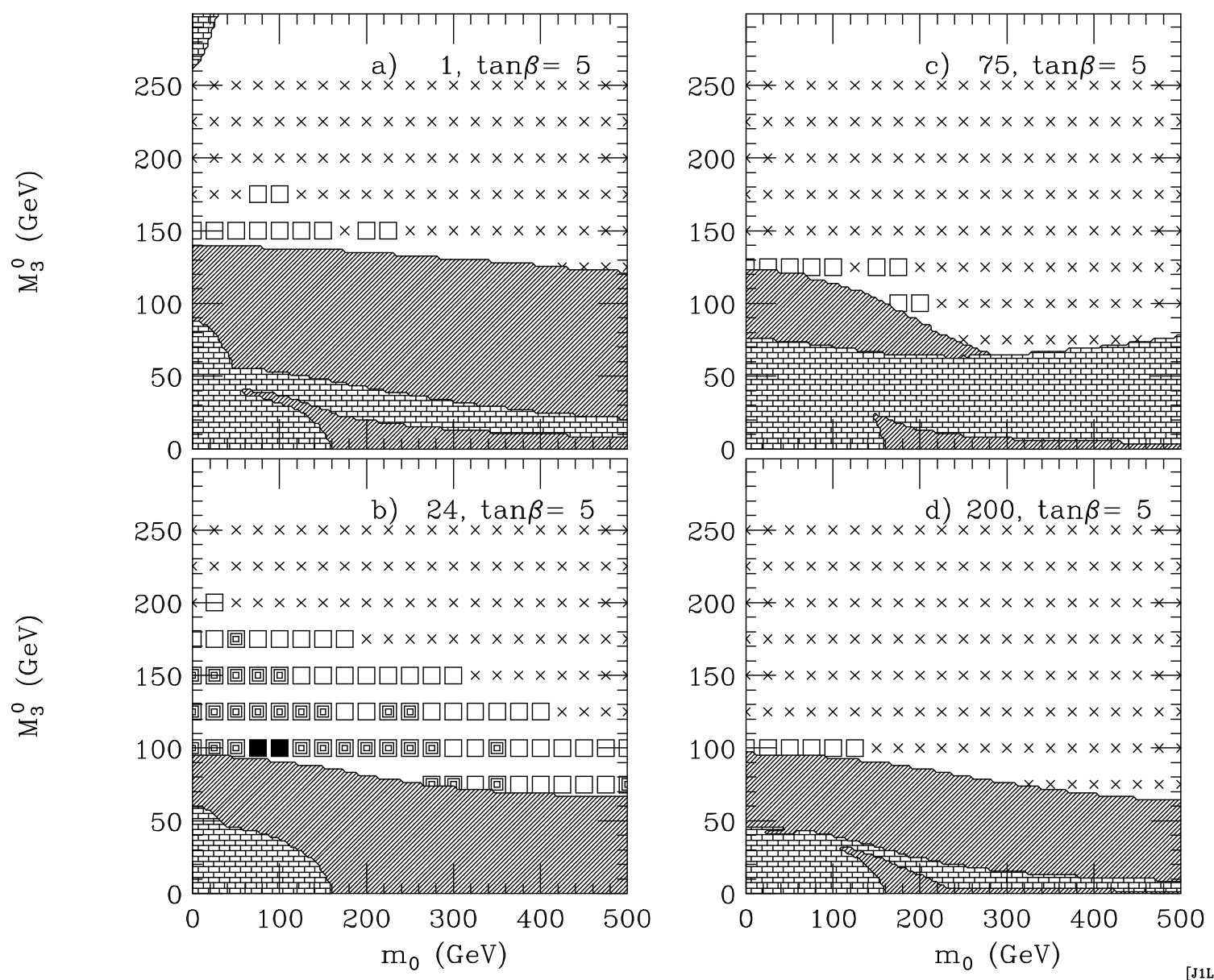

FIG. 8. The same as Fig. 6, except for the J1L signal. 

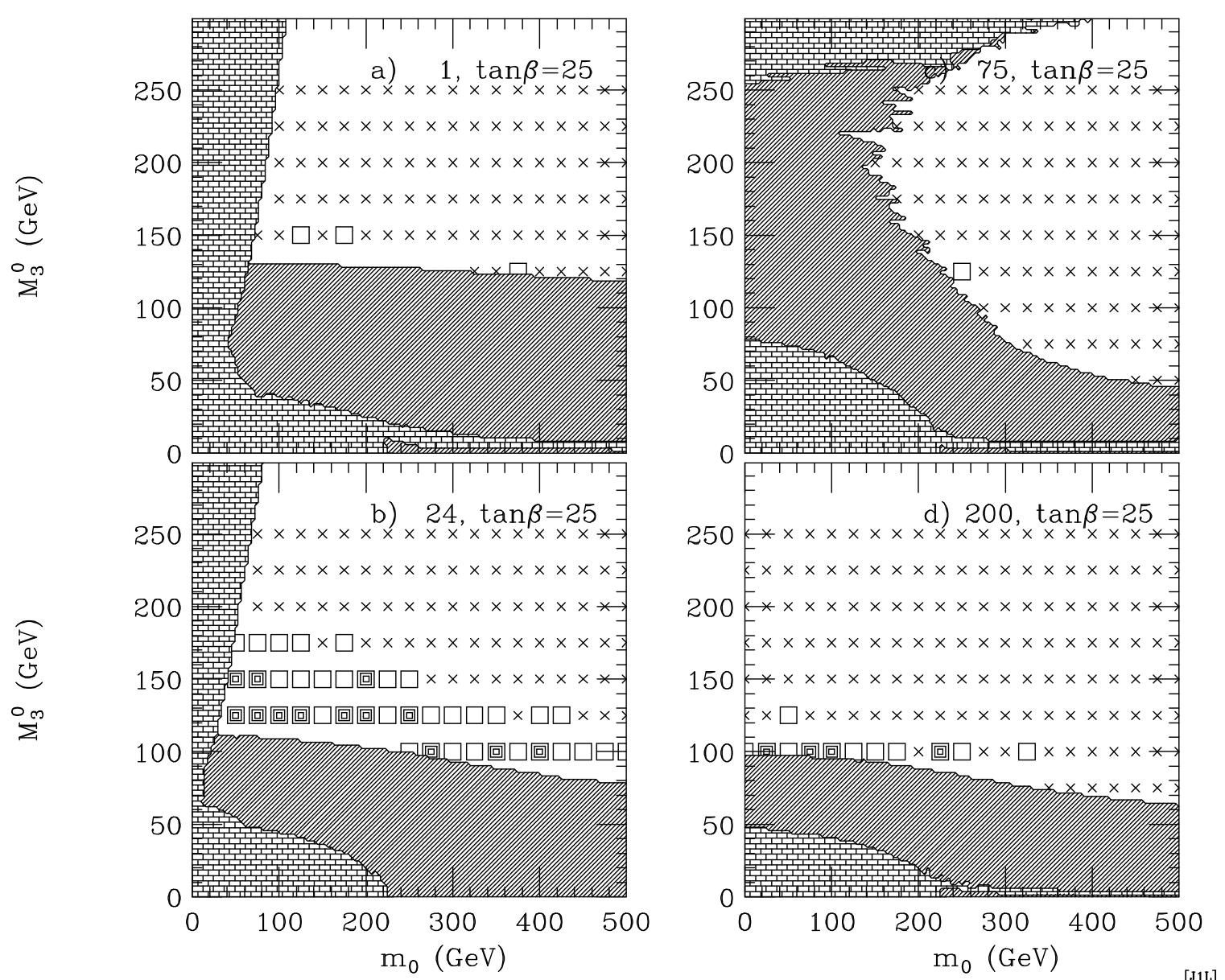

FIG. 9. The same as Fig. 7, except for the J1L signal. 

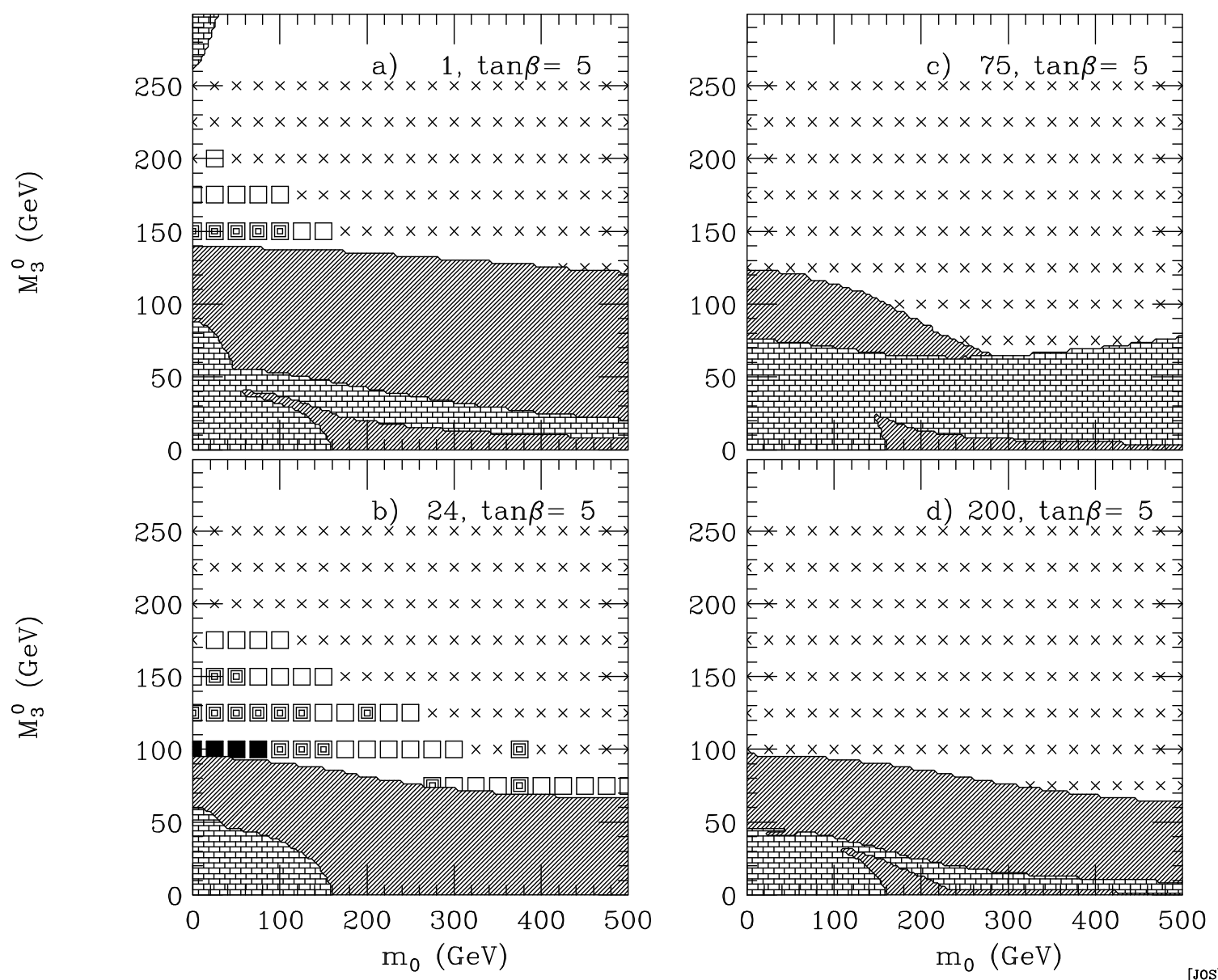

FIG. 10. The same as Fig. 6, except for the JOS signal. 

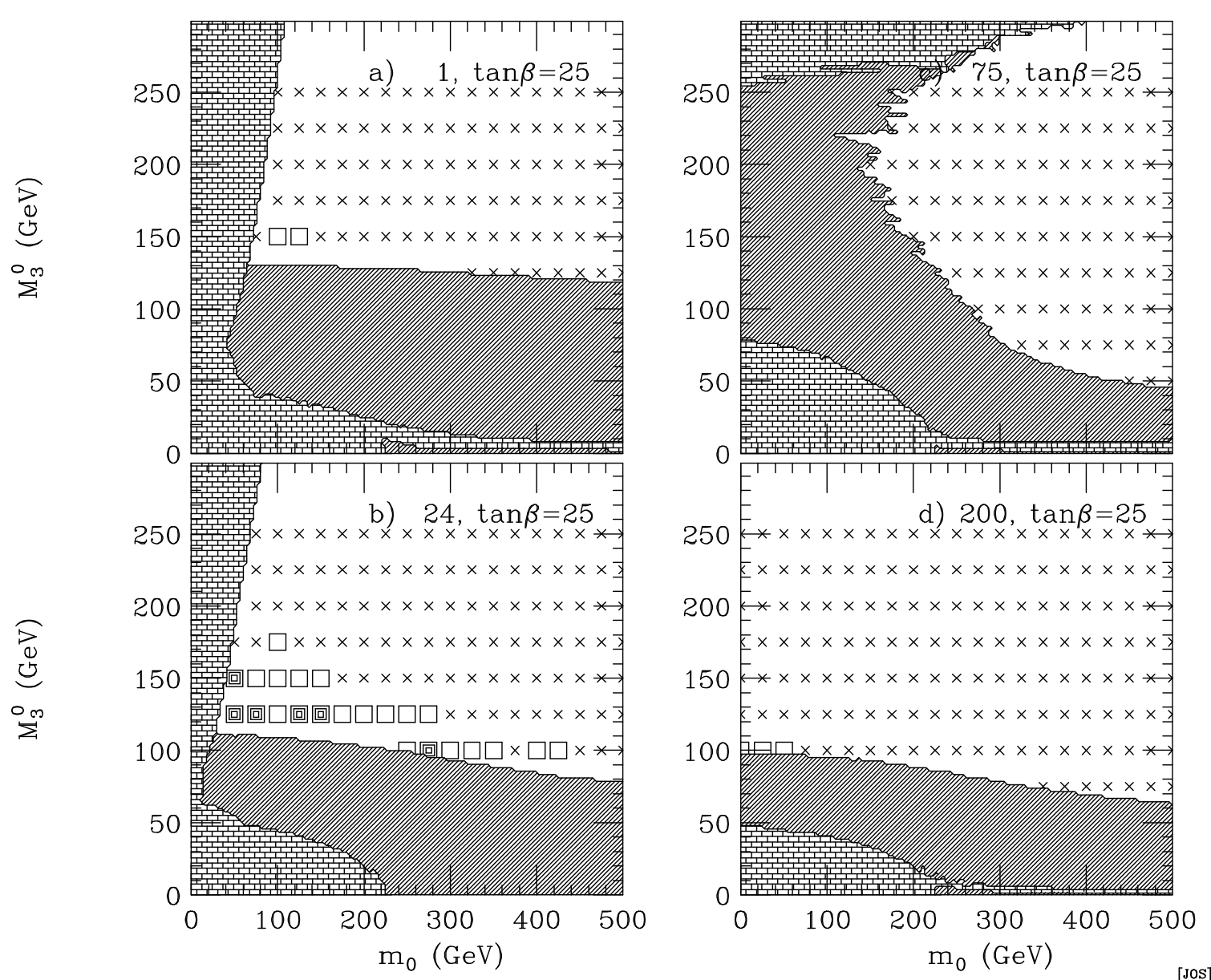

FIG. 11. The same as Fig. 0, except for the JOS signal. 

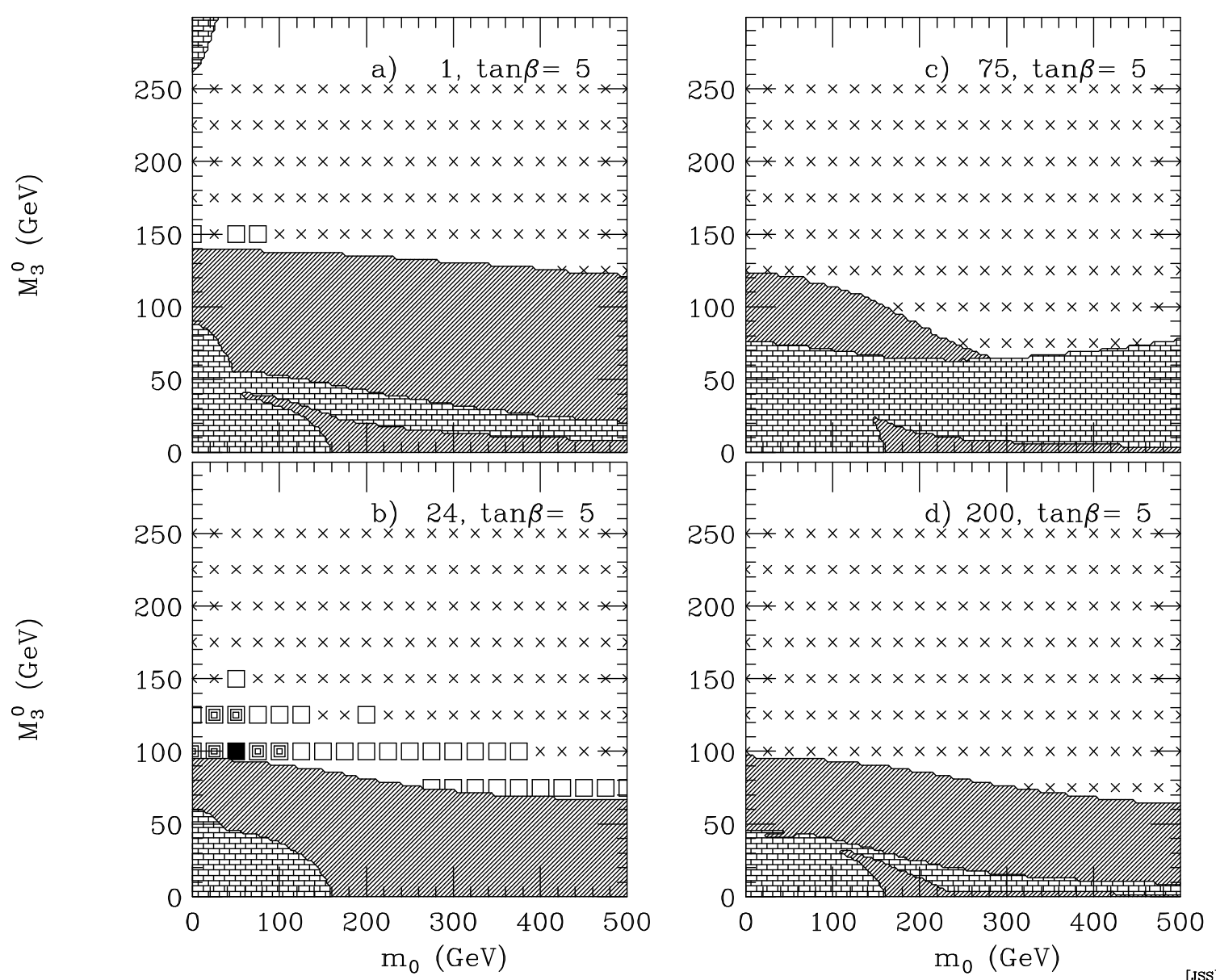

FIG. 12. The same as Fig. 6, except for the JSS signal. 

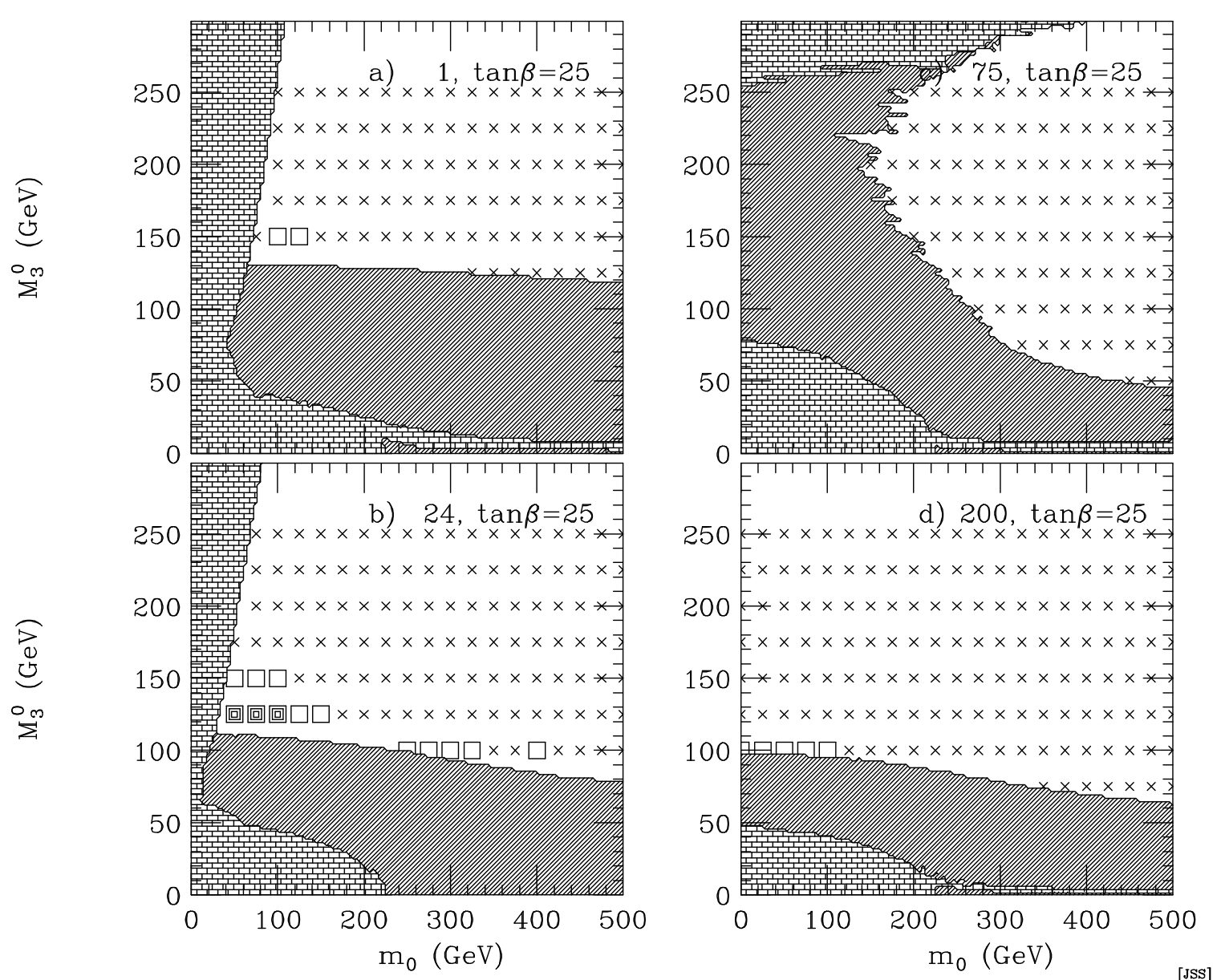

FIG. 13. The same as Fig. 7, except for the JSS signal. 

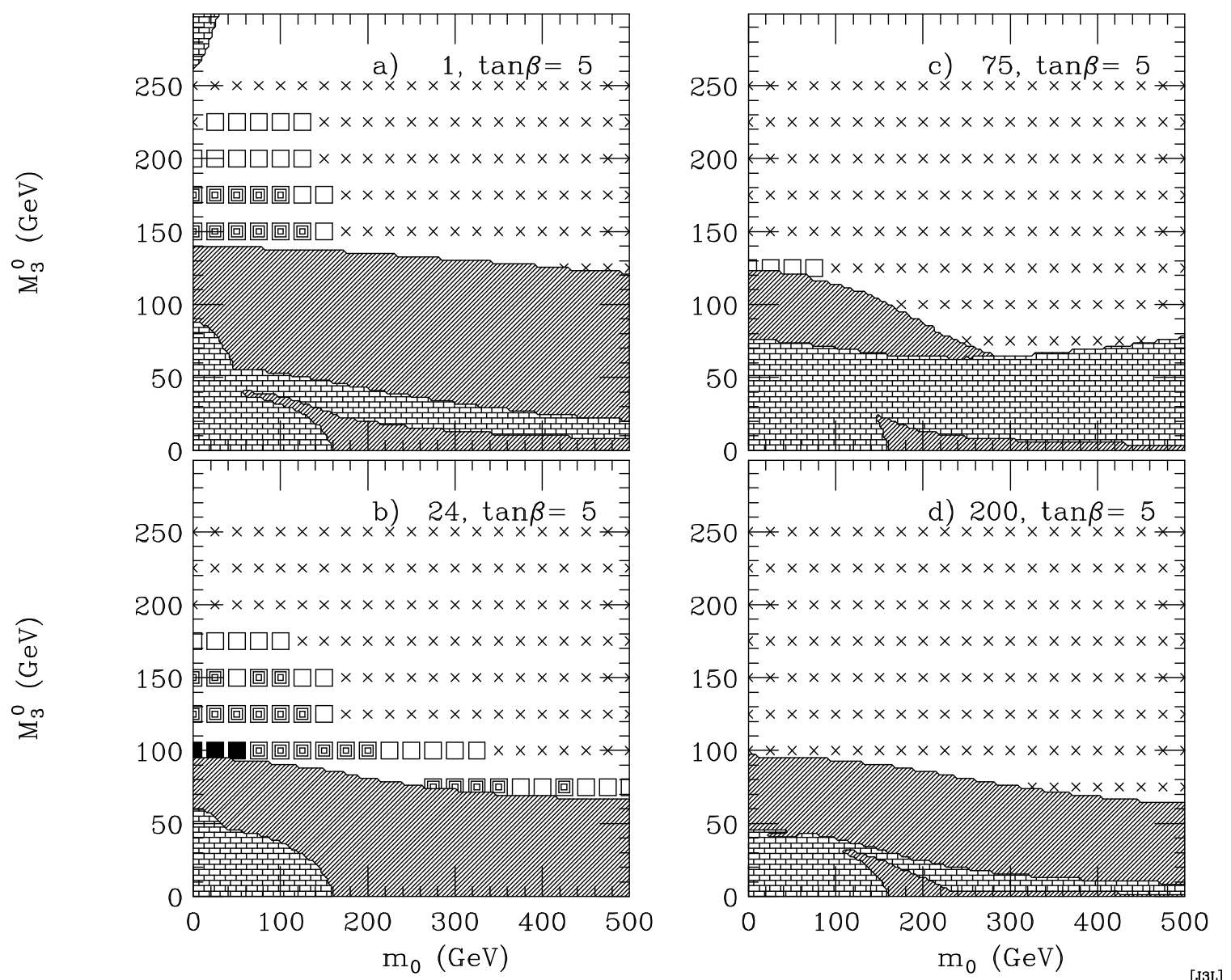

FIG. 14. The same as Fig. 6, except for the J3L signal. 

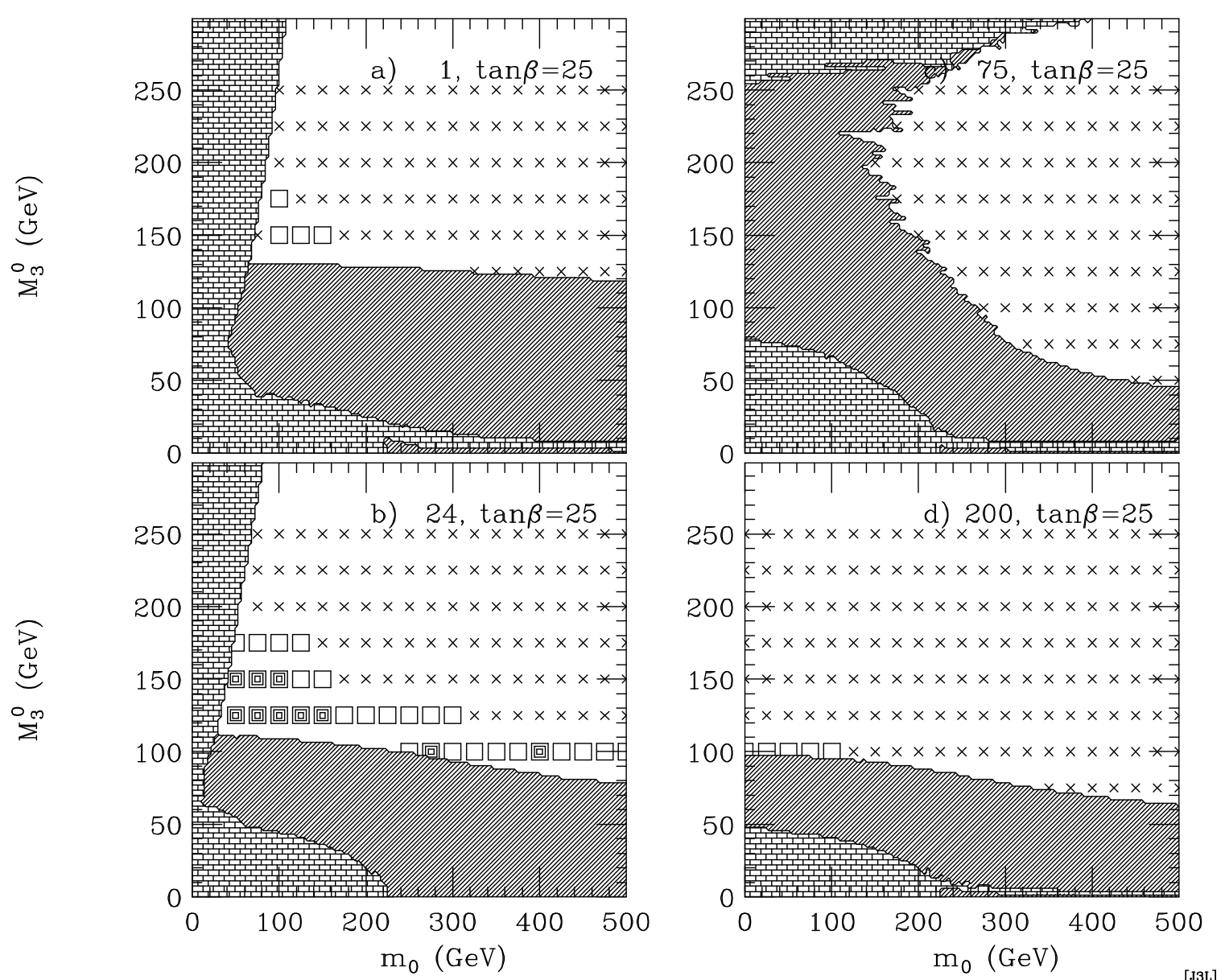

FIG. 15. The same as Fig. [, except for the J3L signal. 

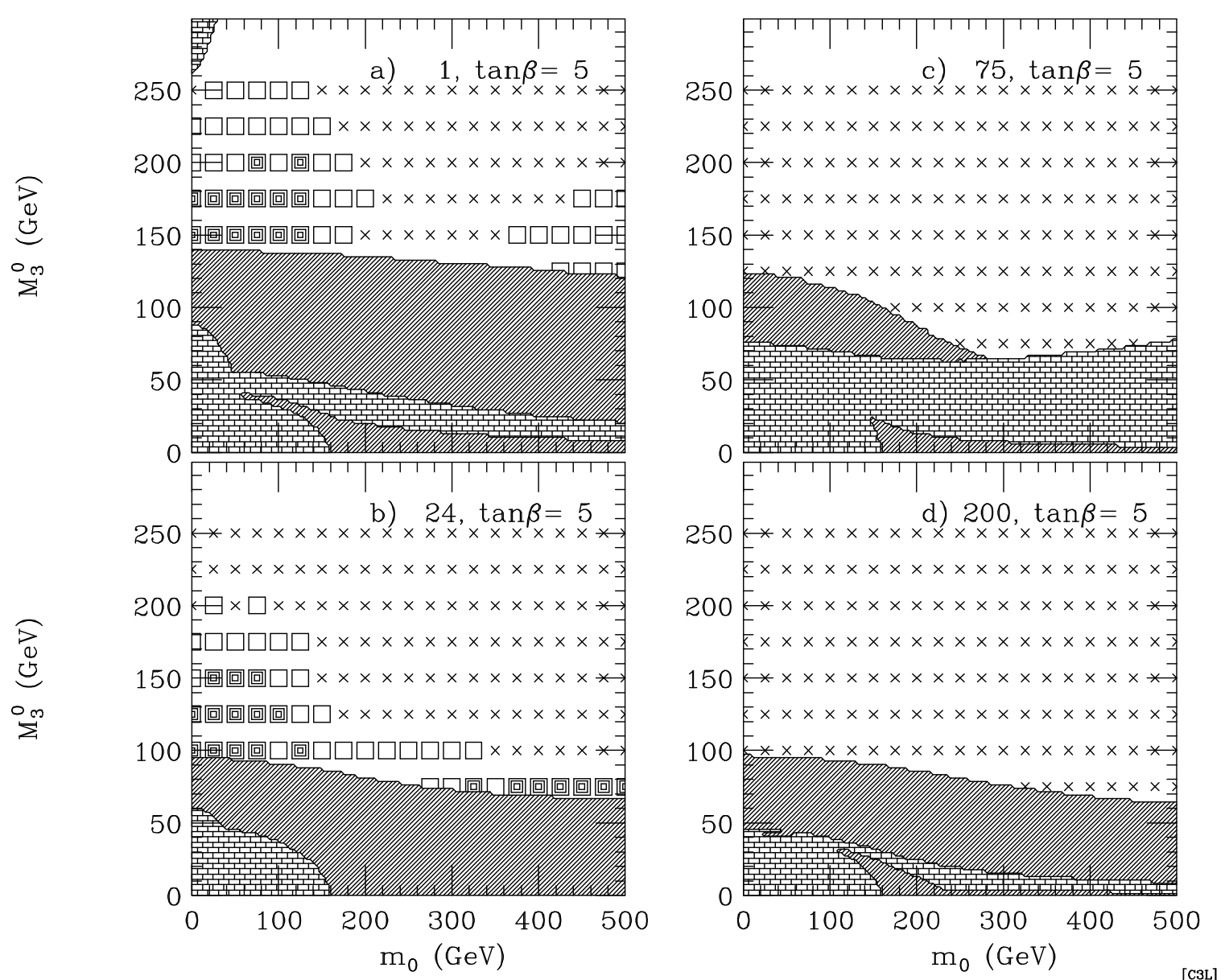

FIG. 16. The same as Fig. 6, except for the C3L signal. 

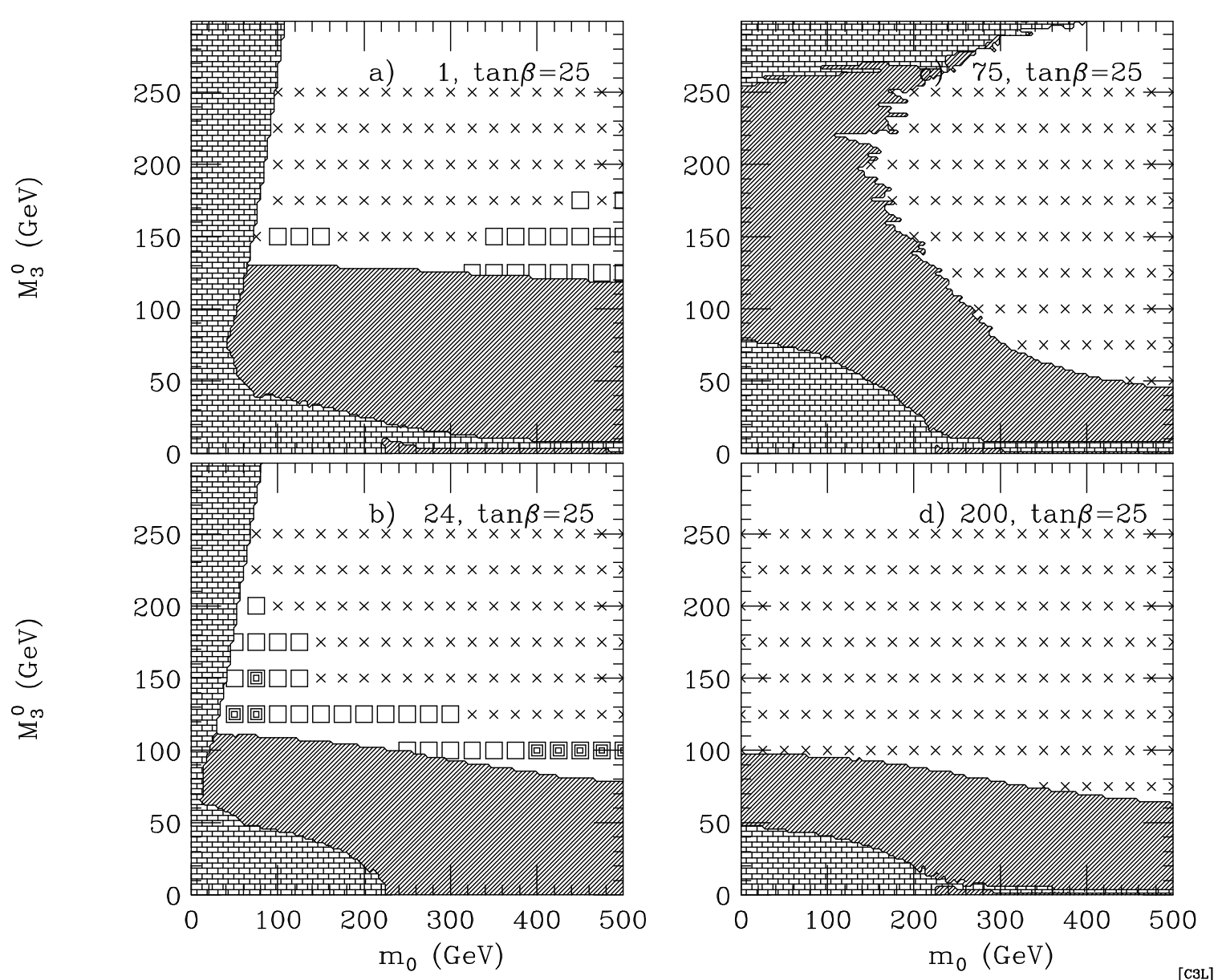

FIG. 17. The same as Fig. 07, except for the C3L signal. 

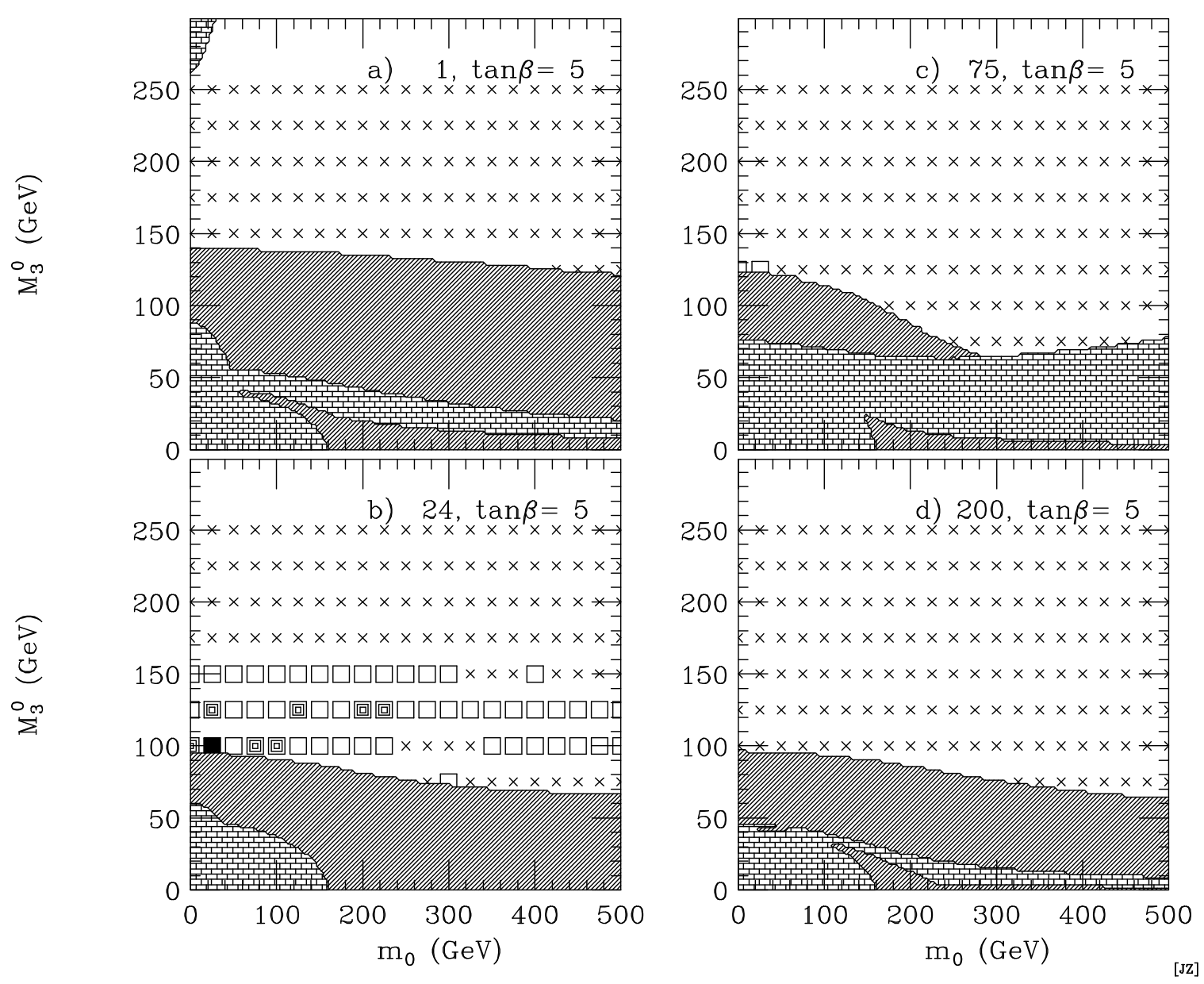

FIG. 18. The same as Fig. 6, except for the JZ signal. 

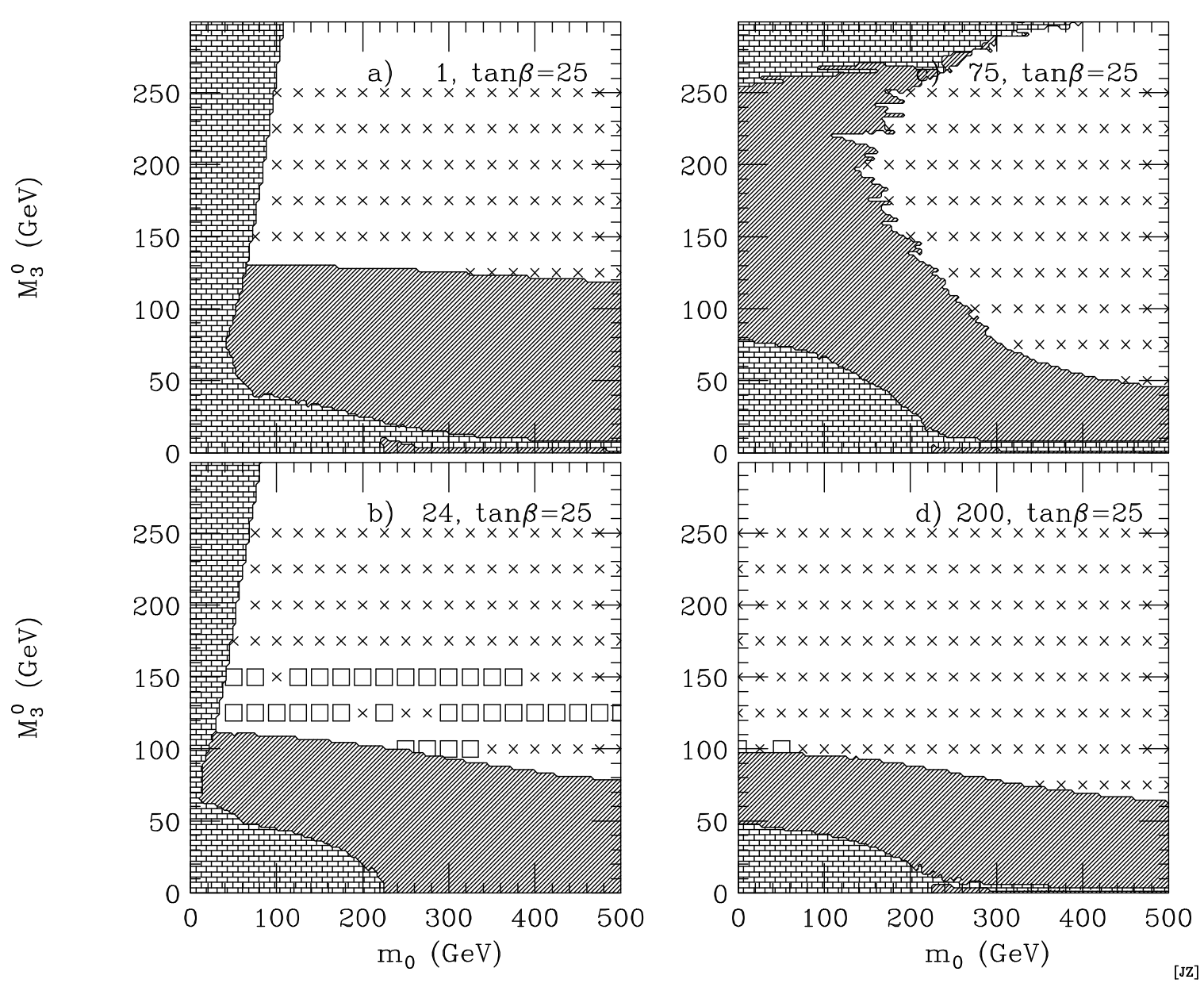

FIG. 19. The same as Fig. 7, except for the JZ signal. 

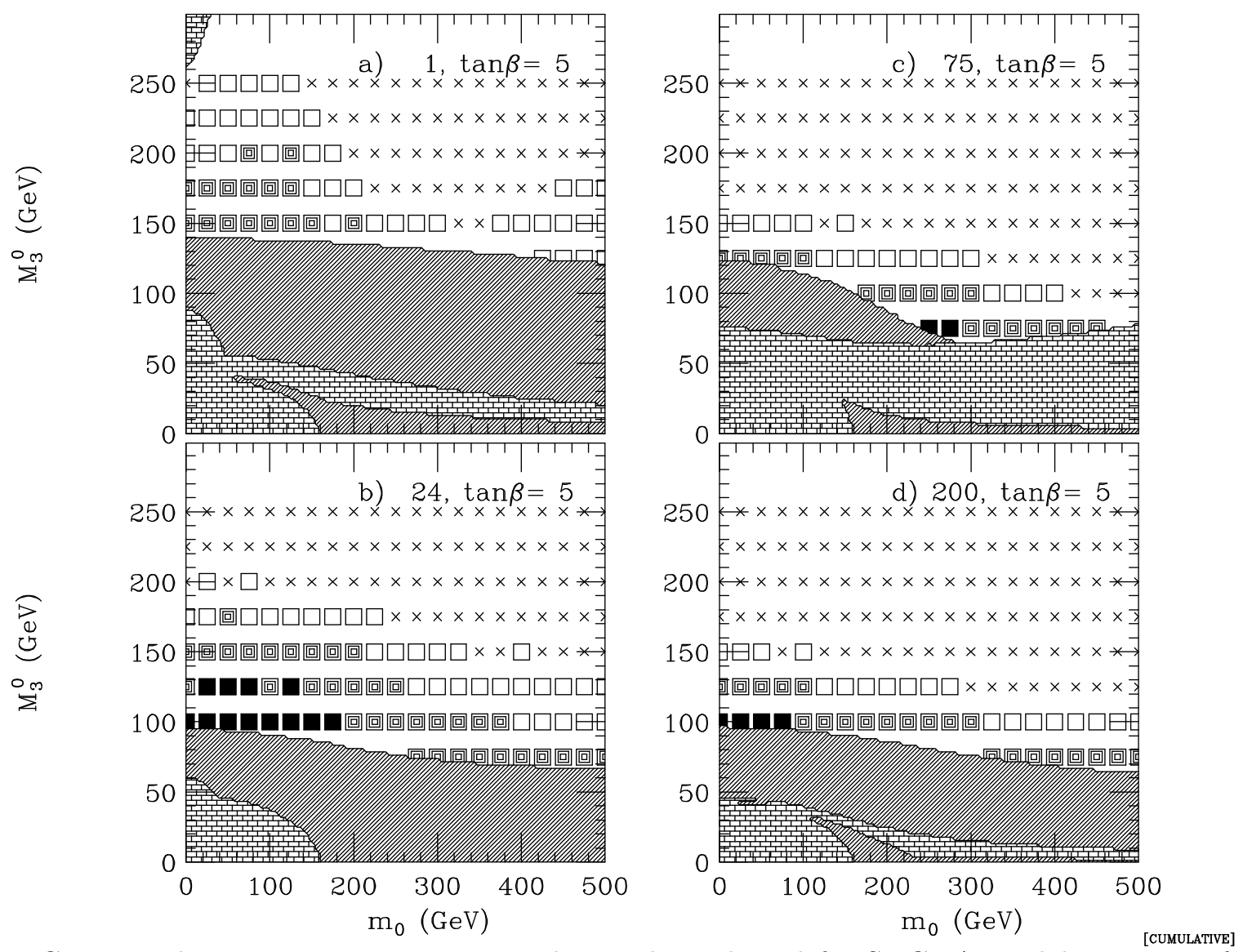

FIG. 20. The same as Fig. 6, except the reach is plotted for SUGRA models via any of the signals considered in this paper. 

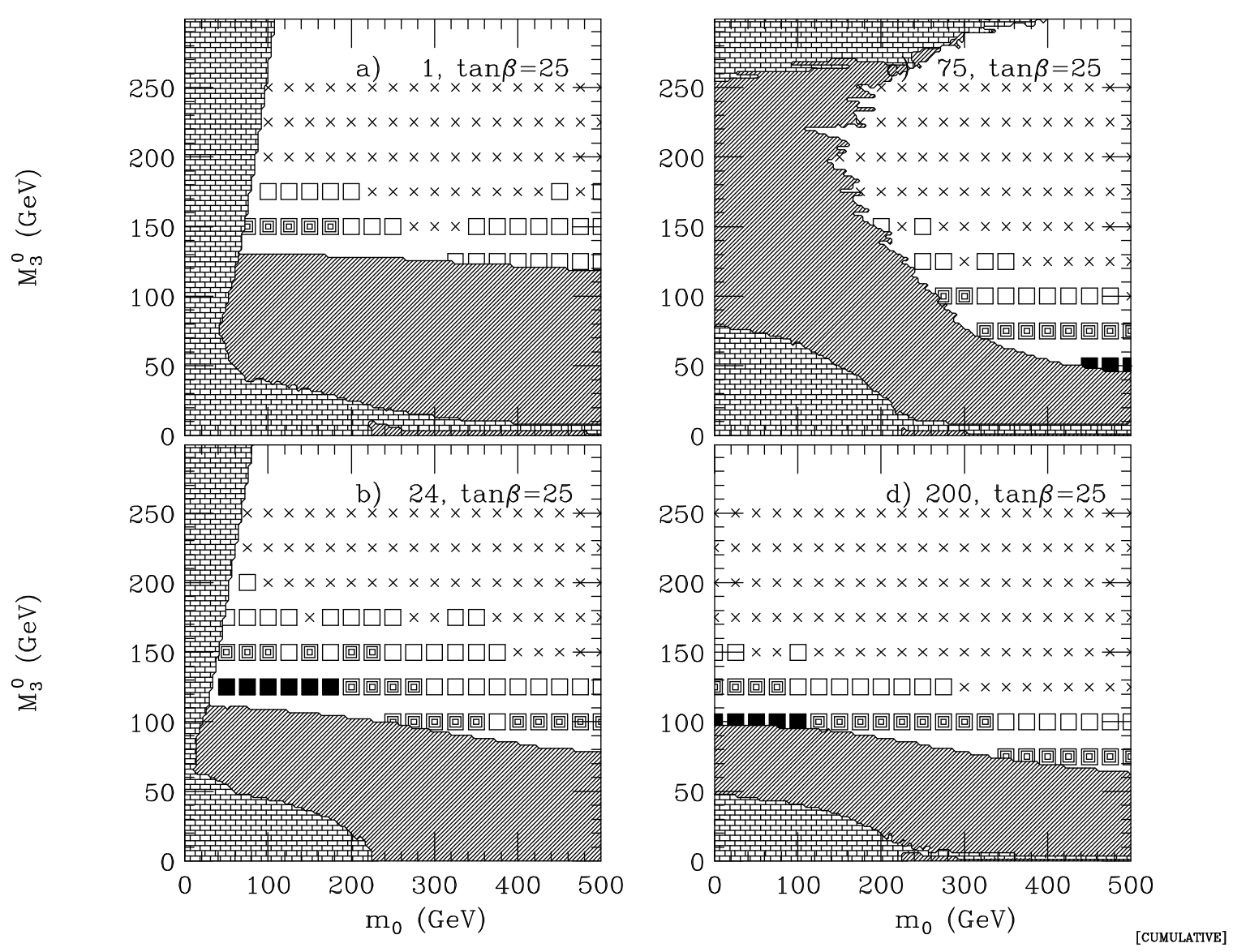

FIG. 21. The same as Fig. 20, except for $\tan \beta=25$. 